\title{
Laminar flow in three-dimensional square-square expansions
}

\author{
P.C. Sousa ${ }^{a}$, P.M. Coelho ${ }^{\text {b }}$, M.S.N. Oliveira ${ }^{a}$, M.A. Alves ${ }^{a}{ }^{a}$ \\ a Departamento de Engenharia Química, CEFT, Faculdade de Engenharia da Universidade do Porto, Rua Dr. Roberto Frias, $4200-465$ Porto, Portugal \\ b Departamento de Engenharia Mecânica, CEFT, Faculdade de Engenharia da Universidade do Porto, Rua Dr. Roberto Frias, 4200-465 Porto, Portugal
}

\section{A R T I C L E I N F O}

\section{Article history:}

Received 25 May 2010

Received in revised form 2 June 2011

Accepted 3 June 2011

Available online 13 June 2011

\section{Keywords:}

Viscoelastic fluid

Boger fluid

Flow visualization

PIV

3D expansion flow

Numerical simulations

\begin{abstract}
A B S T R A C T
In this work we investigate the three-dimensional laminar flow of Newtonian and viscoelastic fluids through square-square expansions. The experimental results obtained in this simple geometry provide useful data for benchmarking purposes in complex three-dimensional flows. Visualizations of the flow patterns were performed using streak photography, the velocity field of the flow was measured in detail using particle image velocimetry and additionally, pressure drop measurements were carried out. The Newtonian fluid flow was investigated for the expansion ratios of 1:2.4, 1:4 and 1:8 and the experimental results were compared with numerical predictions. For all expansion ratios studied, a corner vortex is observed downstream of the expansion and an increase of the flow inertia leads to an enhancement of the vortex size. Good agreement is found between experimental and numerical results. The flow of the two non-Newtonian fluids was investigated experimentally for expansion ratios of 1:2.4, 1:4, 1:8 and $1: 12$, and compared with numerical simulations using the Oldroyd-B, FENE-MCR and SPTT constitutive equations. For both the Boger and shear-thinning viscoelastic fluids, a corner vortex appears downstream of the expansion, which decreases in size and strength when the elasticity of the flow is increased. For all fluids and expansion ratios studied, the recirculations that are formed downstream of the square-square expansion exhibit a three-dimensional structure evidenced by a helical flow, which is also predicted in the numerical simulations.
\end{abstract}

(c) 2011 Elsevier B.V. All rights reserved.

\section{Introduction}

The flow of Newtonian and non-Newtonian fluids in channels with variable cross-section, which include contractions and expansions, is a classical fluid mechanics problem, which has been addressed in a number of experimental and numerical studies published in the literature (e.g. [1-7]). The investigation of these flows is essential not only from a fundamental point of view but also due to their importance in practical applications, namely in the polymer processing industry. The flow through sudden contractions is a benchmark problem that has received broader attention than the flow through abrupt expansions, possibly due to the wide and interesting nature of the flow patterns that occur in contraction flows, particularly with viscoelastic fluids (e.g. vortex enhancement, divergent and unsteady flow) [8], and its applicability to estimate the extensional viscosity in entry-flows (e.g. $[9,10])$. Nevertheless, expansion flows can also be used to understand entry-flow problems and to validate numerical codes.

Early experimental studies on expansion flows focused on Newtonian fluids in two-dimensional (2D) geometries [11-13]. It was found that Moffatt vortices [14] appear downstream of the

\footnotetext{
* Corresponding author. Tel.: +351 225081680; fax: +351 225081449.

E-mail addresses: psousa@fe.up.pt (P.C. Sousa), pmc@fe.up.pt (P.M. Coelho), monica.oliveira@fe.up.pt (M.S.N. Oliveira),mmalves@fe.up.pt (M.A. Alves).
}

expansion and that flow inertia promotes the enhancement of these recirculations. Later on, Acrivos and Schrader [15] and Milos et al. [16] investigated numerically the flow of Newtonian fluids at high values of the Reynolds number $(R e)$ and they found that above a critical value of Re the flow becomes unsteady. Furthermore, according to the experiments of Townsend and Walters [17] and the numerical predictions of Baloch et al. [18], a pair of lip vortices develops close to the re-entrant corner for high expansion ratios. These vortices then expand to the downstream wall increasing in size with the Reynolds number.

Townsend and Walters [17] have also studied the flow behavior of viscoelastic fluids through expansions, including the flow of a $0.15 \%$ aqueous solution of polyacrylamide (PAA) through 3:40 and $1: 80$ planar expansions, and the flow of a $0.1 \%$ aqueous solution of xanthan gum and glass fibers through three-dimensional (3D) axisymmetric expansions (expansion ratio of 3:40). For viscoelastic fluids, the recirculations also appear downstream of the expansion, but unlike the Newtonian case, the recirculations decrease in size when the elasticity of the flow is increased. Baloch et al. [19] used the linear form of the Phan-Thien and Tanner (PTT) model [20,21] to numerically simulate a number of viscoelastic flows in planar (expansion ratio of 3:40 and 1:80) and 3D (expansion ratio of $3: 3: 40$ ) geometries. In agreement with the experimental work of Townsend and Walters [17], the numerical results of Baloch et al. [19] reported a decrease in the vortex 
activity due to viscoelasticity, which pushes the recirculations against the downstream corners as previously described by Halmos and Boger [22]. In particular, the viscoelastic fluid flow through planar expansions shows a reduction of the vortex size and strength as the Deborah number $(D e)$ is increased. Eventually, at high Deborah number flows, the recirculations downstream of the expansion seem to disappear altogether. This suppression mechanism has been compared to the extrudate-swell phenomenon, in which the polymer molecules passing through an expansion and entering a larger cross-section reservoir relax the elastic stresses by expanding the main flow stream tube [23]. Another interesting feature worth mentioning is the onset of a lip vortex in the inlet channel upstream of the expansion observed for Upper-Convected Maxwell (UCM) and Oldroyd-B fluids flowing through a 1:3 planar expansion under creeping flow conditions [23]. More recently, the same authors investigated the effect of the expansion ratio on the flow patterns of a UCM fluid [24]. For the lower expansion ratios, a non-monotonic variation of vortex length with $D e$ was predicted, with vortex reduction at low $D e$, followed by vortex enhancement at higher $D e$. For high expansion ratios (ER $>3$ ), the vortex size was found to decrease monotonically as De increases.

There are several works that report the development of asymmetric flow in planar expansions for Newtonian and non-Newtonian fluids (e.g. [25-31]). Cherdron et al. [25] used flow visualization and laser-Doppler anemometry techniques to quantify the steady asymmetry that develops for the Newtonian flow in symmetric planar sudden-expansion geometries, and concluded that the intensity of fluctuating energy measured in such low Reynolds number flows can even be larger than that observed in corresponding turbulent flows. Fearn et al. [26] investigated experimental and numerically the Newtonian fluid flow through a symmetric sudden planar expansion $(E R=3)$ and found that the flow becomes asymmetric and time-dependent for high Reynolds numbers, and related this effect with the 3D structure that develops under those conditions. For generalized Newtonian fluids there are also a number of works that investigate the effect of shearthinning and shear-thickening effects on the critical conditions for development of flow asymmetries in planar sudden expansion flows. Manica and Bortoli [27] used a power-law model to study numerically the laminar flow in a 1:3 planar expansion, and concluded that for shear-thinning fluids the critical Reynolds number for onset of steady flow asymmetry increases as the power law in$\operatorname{dex}(n)$ is reduced, while the opposite happens to shear-thickening fluids as $n$ increases. More recently, Neofytou [28] used Casson and power-law rheological models to investigate the laminar flow in a 1:2 planar expansion. For both non-Newtonian fluids the numerical results showed a linear relation between the inverse dimensionless inlet wall shear stress, at the critical flow conditions for onset of asymmetry flow, and the corresponding dimensionless wall shear rate for the range of Power-Law index and Bingham numbers investigated.

There are also a few studies investigating the stabilizing effect of viscoelasticity on the critical conditions for onset of flow asymmetries in planar expansion flows. Oliveira [29] studied numerically the flow of constant shear viscosity viscoelastic fluids (Boger fluids) through 1:3 planar expansions. The working fluids were a viscoelastic fluid that was described using the modified FENE-CR constitutive equation and a Newtonian fluid that was used with the purpose of validating the method and for comparison purposes. The numerical results showed that the flow becomes asymmetric for both fluids, however, for the viscoelastic case, the elasticity tends to stabilize the flow and the critical Reynolds number is higher than that found for a Newtonian fluid. More recently, Rocha et al. [30] also investigated the onset of asymmetries in viscoelastic fluid flow through a 1:4 planar sudden expansion. The authors determined the critical Re at which the bifurcation phenomenon occurs, and concluded that it is lower than that obtained for lower expansion ratios [29]. Asymmetries in the mean axial velocity profile of a shear-thinning fluid flowing through an axisymmetric abrupt expansion were also found in the work of Dales et al. [32] in which the fluid was an aqueous solution of PAA and the flow regime was turbulent. However, in this case, the Newtonian fluid (water) flow did not show any asymmetries. More recently, another experimental study [33] showed that for a 1:2 axisymmetric expansion the Newtonian fluid flow also becomes asymmetric (and steady), under laminar flow conditions, above $R e=1139$. For $R e>1400$ the flow eventually becomes timedependent.

In most of the planar expansion flows discussed above, threedimensional effects are negligible and less demanding 2D numerical simulations can be used to predict the flow correctly. Configurations where three-dimensional effects are important are more challenging to analyze experimental and numerically and as such, the number of published works concerning this topic is reduced. Two important exceptions are the works of Burgos and Alexandrou [34] and Alexandrou et al. [35] in which the flow of a generalized non-Newtonian fluid (Herschel-Bulkley) through three-dimensional 1:2 and 1:4 expansions was investigated. In the first work [34], the unsteady flow in a 1:2 sudden expansion was studied and in the latter [35], the steady flow of Herschel-Bulkley fluids was investigated. The authors showed that the development of yielded and unyielded regions in this type of 3D geometries depends on the expansion ratio and on Bingham and Reynolds numbers.

In this work, we focus on the flow through a three-dimensional square-square expansion, with different expansion ratios (ER $=2.4$, 4,8 and 12, defined as the ratio between the side lengths of the downstream and upstream square ducts). We use Newtonian and viscoelastic fluids with different rheological characteristics: a Boger fluid, for which the shear viscosity is nearly independent of the shear rate and a shear-thinning viscoelastic fluid. Visualizations were undertaken in order to assess the flow patterns and detailed particle image velocimetry (PIV) measurements were performed to quantify the velocity field. For the Boger fluid, pressure drop measurements are also presented and discussed. Moreover, 3D numerical simulations were carried out in order to predict the Newtonian and non-Newtonian fluid flow through squaresquare expansions.

\section{Experimental techniques}

\subsection{Experimental set-up}

A scheme of the experimental set-up is shown in Fig. 1. The main test section in the experimental rig has a square cross-section of length $L=1.75 \mathrm{~m}$, which is composed of a downstream square channel, with a side length of $2 \mathrm{H}_{2}=24.0 \mathrm{~mm}$, and an upstream interchangeable square channel which fits inside the larger one and has a smaller side length. The internal side length of the inner square part can be set to $2 H_{1}=10.0,6.0,3.0$ or $2.0 \mathrm{~mm}$ in order to obtain the desired expansion ratios, ER $=H_{2} / H_{1}=2.4,4,8$ or 12 .

The upstream and downstream sections are denoted by subscripts 1 and 2, respectively. For optical access, the channels are made of transparent perspex.

The flow rate is set by adjusting the difference between the liquid levels in the two reservoirs, applying vacuum to the left-hand side reservoir using a vacuum pump (KNF Loboport N811-KT.18) and selecting the diameter of the pipe that connects the channel to the outflow reservoir. We do not use valves to regulate the flow rate to avoid degradation of the viscoelastic fluids in the narrow 


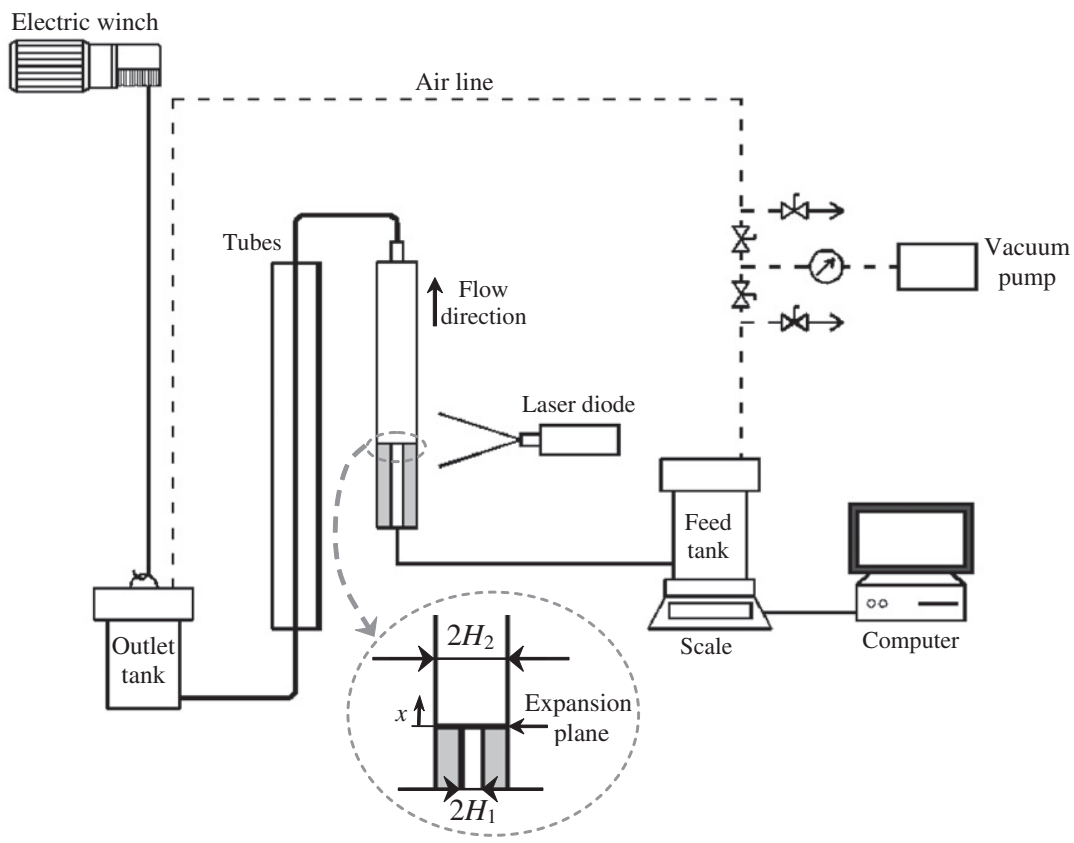

Fig. 1. Schematic diagram of the experimental set-up.

passage of the valve. The right-hand side reservoir was used to supply the fluid that flowed through the column, and it was placed on a weighing scale (KERN DS 16k0.2; with readout of $0.2 \mathrm{~g}$ and maximum range of $16 \mathrm{~kg}$ ) in order to measure the mass flow rate $\left(Q_{m} \equiv \Delta\right.$ Weight/ $\Delta$ time). More details of the experimental set-up can be found in Ref. [8].

\subsection{Measurement techniques}

Two different optical techniques were used in this study, in order to characterize the flow field: streak photography and particle image velocimetry. For this purpose, the fluids used in the experiments were seeded with $10 \mu \mathrm{m}$ PVC tracer particles.

Streak photography uses long time exposures to obtain a visual fingerprint of the flow patterns. For flow illumination, we used a $635 \mathrm{~nm} 5 \mathrm{~mW}$ laser diode (Vector, model $5200-20$ ) or a $532 \mathrm{~nm}$ $3 \mathrm{~mW}$ laser diode (Imatronic, model LLM115). In both cases a cylindrical lens is attached to the diode to generate a sheet of light. The streak images were captured using a digital camera (Canon EOS $30 \mathrm{D}$ ) with a macro lens (Canon EF100 mm, f/2.8), placed perpendicularly to the light sheet.

For the measurement of the velocity field, a doubled pulsed Nd:YAG laser, with a maximum energy of $50 \mathrm{~mJ}$ (Solo PIV III from New Wave Research) was used. The laser was combined with appropriate optical components, producing a planar light sheet that illuminates the plane under study. The images were recorded using a digital CCD camera (Flow Sense $2 \mathrm{M}$ from Dantec Dynamics equipped with a Nikon AF Micro Nikkor $60 \mathrm{~mm}$ f/2.8D lens) which was placed perpendicularly to the light sheet. The images were processed using FlowManager v4.60 software (Dantec Dynamics) and the velocity vector map for each pair of images was determined using a cross-correlation technique. A detailed discussion of the technique employed can be found in Ref. [36].

The flow visualizations and the velocity field measurements using PIV were both undertaken at different parallel planes of the flow in the square channel. Therefore, it was necessary to translate simultaneously the light source and the camera utilized for each optical technique using for this purpose a $x-y$ traverse and a dial comparator (readout of $\pm 0.01 \mathrm{~mm}$ ).
Pressure drop measurements across the expansion were carried out using differential pressure sensors (Honeywell, model 26PC series), previously calibrated using a hydrostatic column of water, which are able to cover pressure differences up to $6.9 \mathrm{kPa}$. The experiments were performed by determining the pressure drop $(\Delta p)$ across the expansion, with one pressure port located upstream $\left(p_{1}\right)$ and another port $\left(p_{2}\right)$ located downstream of the expansion plane. The precise locations of the pressure ports are listed in Table 1 for different ER.

For each flow rate studied, the output signal of the pressure transducer was recorded using LabView v7.1 software, until the steady-state was reached.

\subsection{Rheological characterization}

A Newtonian and two different non-Newtonian fluids were used in the experiments. Table 2 summarizes the composition and density $(\rho)$ of the fluids, measured at $293.2 \mathrm{~K}$ using two hydrometers (readability of $1 \mathrm{~kg} / \mathrm{m}^{3}$; ranges $1100-1200 \mathrm{~kg} / \mathrm{m}^{3}$ and $1200-1300 \mathrm{~kg} / \mathrm{m}^{3}$ ). A biocide (Kathon LXE, Rohm and Haas) was added to the solutions, at a weight concentration of $25 \mathrm{ppm}$, in order to minimize bacterial growth and biological degradation of the fluids.

Furthermore, the working fluids were characterized rheologically at different temperatures in the range of $283.2 \leqslant T$ / $\mathrm{K} \leqslant 303.2$ using a shear rheometer (MCR301, Anton Paar) and the time-temperature superposition method was used to obtain a master flow curve.

The variation of the viscosity with temperature can be described using an Arrhenius equation [37],

Table 1

Pressure port locations in the $x$-direction (streamwise) for each expansion ratio. The expansion plane is located at $x=0$.

\begin{tabular}{lll}
\hline & $x_{1} / \mathrm{mm}$ & $x_{2} / \mathrm{mm}$ \\
\hline $\mathrm{ER}=4$ & -99 & 152 \\
$\mathrm{ER}=8$ & -48 & 153 \\
$\mathrm{ER}=12$ & -29 & 154 \\
\hline
\end{tabular}


Table 2

Composition by weight and density of the fluids measured at $293.2 \mathrm{~K}$.

\begin{tabular}{|c|c|c|c|c|c|c|}
\hline Fluid & PAA [ppm] & Glycerin [\%] & Water [\%] & Kathon [ppm] & $\mathrm{NaCl}[\%]$ & $\rho\left[\mathrm{kg} \mathrm{m}^{-3}\right]$ \\
\hline Newtonian & - & 84.99 & 15.01 & 25 & - & 1221 \\
\hline Boger & 100 & 90.96 & 7.52 & 25 & 1.51 & 1249 \\
\hline Shear-Thinning & 600 & 59.94 & 40.00 & 25 & - & 1156 \\
\hline
\end{tabular}

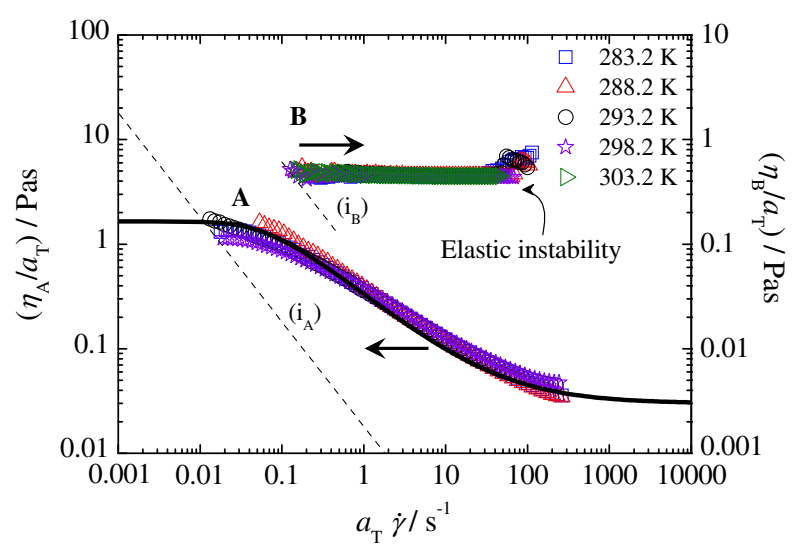

Fig. 2. Master curves of the steady shear viscosity measured at different temperatures for the Boger (B) and shear-thinning (A) fluids (symbols). The solid line represents the fit of the SPTT model to the experimental data of the shear-thinning fluid. The minimum measurable shear viscosity, determined from $20 \times$ the minimum measurable torque of the rheometer, is represented by dashed lines for the shear-thinning fluid $\left(i_{\mathrm{A}}\right)$ and the Boger fluid $\left(i_{\mathrm{B}}\right)$.

$\ln \left(a_{T}\right)=\frac{\Delta H}{R}\left(\frac{1}{T}-\frac{1}{T_{0}}\right)$

where $a_{T}$ is the shift factor, $\Delta H$ is an activation energy, $R$ is the universal gas constant, $T$ is the absolute temperature of the measurement and $T_{0}$ is the absolute reference temperature, which is set here as $T_{0}=293.2 \mathrm{~K}$, the temperature at which the experiments in the square-square expansion were performed. The shift factor is defined as [37],

$a_{T}=\frac{\eta(T)}{\eta\left(T_{0}\right)} \frac{T_{0}}{T} \frac{\rho_{0}}{\rho}$

where $\eta(T)$ and $\rho$ are the shear viscosity and the fluid density at temperature $T$ and $\eta\left(T_{0}\right)$ and $\rho_{0}$ are the shear viscosity and the fluid density at the reference temperature $T_{0}$. Since the range of temperatures of the measurements is small, the shift factor can be simplified to:

$a_{T}=\frac{\eta(T)}{\eta\left(T_{0}\right)}$

For the Newtonian fluid, the shear viscosity at the reference temperature is $\eta\left(T_{0}\right)=0.0982 \mathrm{~Pa}$ and $\Delta H / R=5580 \mathrm{~K}$. For the non-Newtonian fluids we present in Fig. 2 the master curves measured for steady shear flow. For the Boger fluid, we obtained $\Delta H$ / $R=6780 \mathrm{~K}$ for the range of temperatures between 283.2 and $303.2 \mathrm{~K}$.

\section{Numerical method and computational meshes}

The flow through square-square expansions with different expansion ratios ( $E R=2.4,4,8$ and 12 ) was simulated numerically using a fully-implicit finite-volume method with a time marching pressure-correction algorithm [38]. The governing equations that describe an isothermal, laminar and incompressible fluid flow are those of conservation of mass and momentum,

$\nabla \cdot \mathbf{u}=0$

$\rho\left[\frac{\partial \mathbf{u}}{\partial t}+\nabla \cdot \mathbf{u u}\right]=-\nabla p+\eta_{\mathrm{s}} \nabla^{2} \mathbf{u}+\nabla \cdot \tau$

where $\mathbf{u}$ is the velocity vector, $t$ the time, $p$ the pressure and $\eta_{\mathrm{S}}$ the Newtonian solvent viscosity. The extra-stress tensor $\left(\tau_{t}\right)$ is described by the sum of a Newtonian $\left(\boldsymbol{\tau}_{\mathrm{s}}=\eta_{\mathrm{s}}\left(\nabla \mathbf{u}+\nabla \mathbf{u}^{\mathrm{T}}\right)\right)$ and a polymeric solute contribution $(\tau)$. In the numerical simulations of the Newtonian fluid flow, the polymeric contribution to the extra-stress tensor is null $(\nabla \cdot \tau=\mathbf{0})$ and only the Newtonian component remains in Eq. (5). On the other hand, for the numerical simulations of the viscoelastic fluid flow, the polymeric contribution is included and is described using an appropriate rheological constitutive equation. The following general equation was used,

$$
\begin{aligned}
& f(\operatorname{Tr} \tau) \tau+\frac{\lambda}{g(\operatorname{Tr} \tau)}\left(\frac{\partial \tau}{\partial t}+\nabla \cdot \mathbf{u} \tau\right) \\
& \quad=\eta_{\mathrm{P}}\left(\nabla \mathbf{u}+\nabla \mathbf{u}^{\mathrm{T}}\right)+\frac{\lambda}{g(\operatorname{Tr} \tau)}\left(\tau \cdot \nabla \mathbf{u}+\nabla \mathbf{u}^{\mathrm{T}} \cdot \tau\right)
\end{aligned}
$$

where $\lambda$ is the relaxation time, $\eta_{\mathrm{P}}$ is the zero-shear viscosity of the polymer and $f(\operatorname{Tr} \tau)$ and $g(\operatorname{Tr} \tau)$ are functions of the trace of tensor $\tau, \operatorname{Tr}(\tau)$

For the shear-thinning fluid the simplified PTT model was used (hereafter designated as sPTT model), which is obtained setting $g(\operatorname{Tr} \tau)=1$ and considering the linear form for the stress function $f(\operatorname{Tr} \tau)$

$f(\operatorname{Tr} \tau)=1+\frac{\lambda \varepsilon}{\eta_{\mathrm{p}}} \operatorname{Tr}(\tau)$

where $\varepsilon$ is the extensibility parameter. For the Boger fluid two models were used in the numerical simulations, namely the Oldroyd-B model and the FENE-MCR constitutive equation [39,40]. The Oldroyd-B model has the known drawback of predicting an unbounded steady-state extensional viscosity above a critical dimensionless strain rate $\left(\lambda \dot{\varepsilon}_{\text {crit }}=0.5\right)$ and is obtained considering $\varepsilon=0$ (i.e. $f(\operatorname{Tr} \tau)=1)$ and $g(\operatorname{Tr} \tau)=1$ in Eq. (6). The FENE-MCR model is obtained setting $\varepsilon=0$ in Eq. (6), and considering the following stretch function,

$g(\operatorname{Tr} \tau)=\frac{L^{2}+\left(\lambda / \eta_{\mathrm{P}}\right) \operatorname{Tr}(\tau)}{L^{2}-3}$

where the dimensionless parameter $L^{2}$ represents the ratio between the maximum and the equilibrium average dumbbell extensions. For large values of $L^{2}$ the Oldroyd-B model is asymptotically approached, while using finite values of $L^{2}$ removes the unbounded behavior of the steady-state extensional viscosity at large strain rates.

For the shear-thinning fluid flow, the numerical simulations were performed using the SPTT model with one mode and a solvent contribution $[20,21]$. The model parameters were set by fitting the steady shear flow curve obtained experimentally (cf. Fig. 2), using the following parameters [41]: zero-shear polymer viscosity, $\eta_{\mathrm{P}}=1.62$ Pa s; solvent viscosity, $\eta_{\mathrm{S}}=0.03$ Pa s; relaxation time, 
$\lambda=32 \mathrm{~s}$; extensibility parameter, $\varepsilon=0.06$. This fit is also shown in Fig. 2 for comparison with the experimental data.

For the Boger fluid flow, simulations were carried out using both the Oldroyd-B and the FENE-MCR models with the following parameters: $\varepsilon=0, \lambda=3.29 \mathrm{~s}, \eta_{\mathrm{P}}=0.279 \mathrm{~Pa} \mathrm{~s}$ and $\eta_{\mathrm{S}}=0.367 \mathrm{~Pa}$. These parameters were selected based on the fit of a three-mode model with a solvent contribution to the rheological measurements obtained under small amplitude oscillatory shear flow, as detailed in our previous work [8]. For the simulations using the FENE-MCR model a value of $L^{2}=100$ was used (in agreement with other works in the literature, e.g. [40]), although in some cases the influence of $L^{2}$ was also analyzed.

Eqs. (4)-(6) are integrated in space over the computational cells of the mesh and in time over a small time step $(\delta t)$. The time derivative is discretized with an implicit first-order Euler scheme, the diffusive terms are discretized with second-order central differences and the discretization of the advective terms, both in the momentum and constitutive equations, is done using the CUBISTA high-resolution scheme [42].

The computational meshes used in this study for the numerical simulation of the flow through square-square expansions are composed of three-dimensional orthogonal blocks and non-uniform cells that follow a geometrical progression within each direction. For the expansion ratios of $2.4,4$ and 8 , the numerical simulations were carried out using three different meshes: mesh M40 which has 40 cells in $y$ - and $z$-directions of the downstream channel, mesh M64 with 64 cells in $y$ - and $z$-directions and a more refined mesh, M80 which has 80 cells in both orthogonal directions. For the expansion ratio of 12, simulations were performed using three meshes with a slightly different number of cells: mesh M48, M60 and M96, which have 48, 60 and 96 cells in the $y$ - and $z$-directions of the downstream channel, respectively. The total number of cells (NC) and the dimensionless minimum cell size $\left(\Delta x_{\min } / 2 H_{2}, \Delta y_{\min } / 2 H_{2}\right.$ and $\left.\Delta z_{\min } / 2 H_{2}\right)$ of the meshes used for all the ER are presented in Table 3. In Fig. 3 we show mesh M64 used in the numerical simulations of the flow through the $1: 8$ square-square expansion.

In spite of the geometrical symmetry relative to the central planes $(y=0$ and $z=0)$ and diagonal planes $(z= \pm y)$, all simulations were performed on meshes covering the whole wall-to-wall geometries. Regarding the boundary conditions, no-slip condition at the solid walls was imposed and the inlets and outlets were positioned far from the expansion plane so that fully developed flow conditions were enforced. At the outlets, vanishing stream wise gradients of velocity and extra-stress tensor components are imposed, and pressure is linearly extrapolated from the two upstream cell center values.

\section{Table 3}

Characteristics of the computational meshes used (NC: number of computational cells).

\begin{tabular}{clrll}
\hline ER & Mesh & \multicolumn{1}{l}{$\mathrm{NC}$} & $\Delta x_{\min } / 2 \mathrm{H}_{2}$ & $\Delta y_{\min } / 2 \mathrm{H}_{2}=\Delta z_{\min } / 2 H_{2}$ \\
\hline \multirow{2}{*}{2.4} & M40 & 164000 & $2.08 \times 10^{-2}$ & $2.04 \times 10^{-2}$ \\
& M64 & 419840 & $1.39 \times 10^{-2}$ & $1.25 \times 10^{-2}$ \\
& M80 & 656000 & $1.03 \times 10^{-2}$ & $9.93 \times 10^{-3}$ \\
& & & & \\
4 & M40 & 51000 & $1.31 \times 10^{-2}$ & $1.45 \times 10^{-2}$ \\
& M64 & 130560 & $8.20 \times 10^{-3}$ & $8.16 \times 10^{-3}$ \\
& M80 & 408000 & $6.26 \times 10^{-3}$ & $6.25 \times 10^{-3}$ \\
& & & & \\
8 & M40 & 163200 & $7.50 \times 10^{-3}$ & $8.69 \times 10^{-3}$ \\
& M64 & 417792 & $4.69 \times 10^{-3}$ & $4.55 \times 10^{-3}$ \\
& M80 & 652800 & $3.75 \times 10^{-3}$ & $3.75 \times 10^{-3}$ \\
& & & & \\
& M48 & 113664 & $1.08 \times 10^{-2}$ & $9.52 \times 10^{-3}$ \\
& M60 & 177600 & $8.61 \times 10^{-3}$ & $8.33 \times 10^{-3}$ \\
& M96 & 909312 & $1.34 \times 10^{-3}$ & $1.35 \times 10^{-3}$ \\
\hline
\end{tabular}
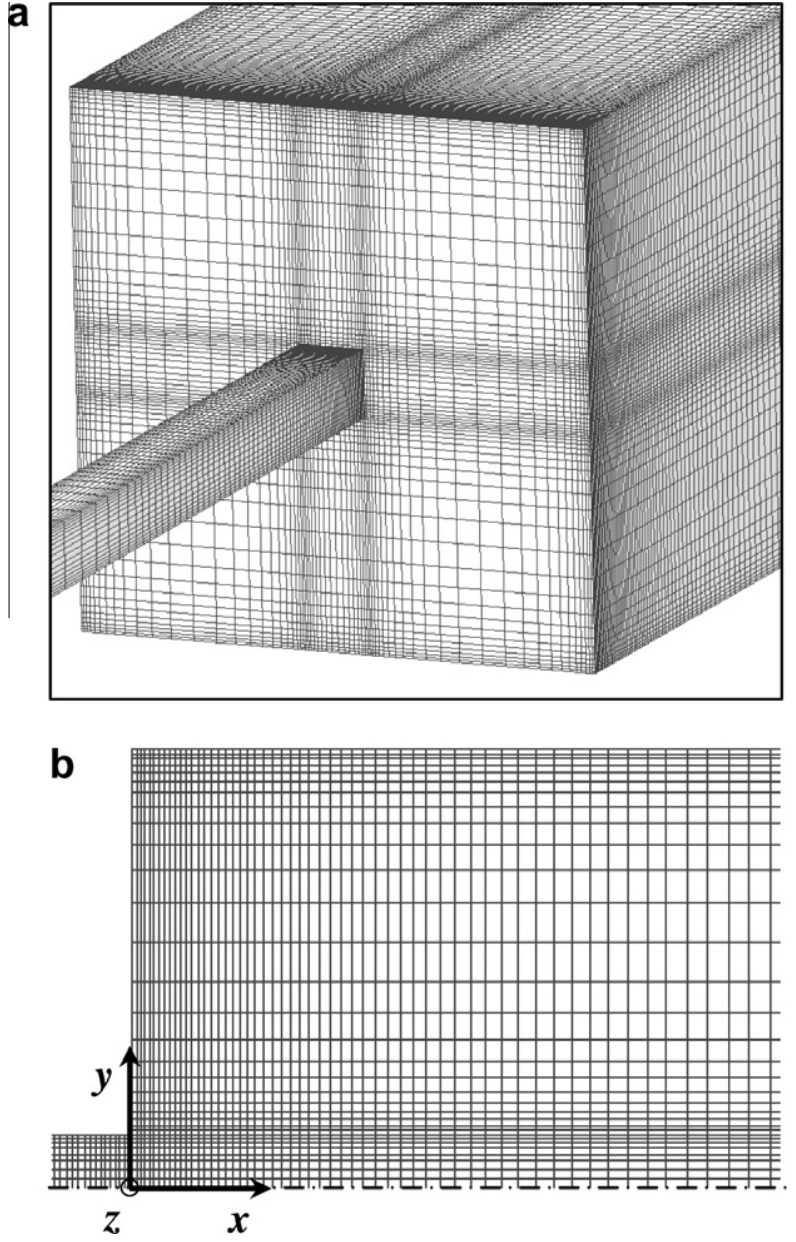

Fig. 3. Zoomed view of mesh M64 used in the numerical simulations of the flow through the 1:8 square-square abrupt expansion. (a) 3D view; (b) $x y$ centerplane $(z=0)$.

\section{Flow patterns and vortex length}

The flow of the Newtonian and viscoelastic fluids through square-square expansions, with different expansion ratios, was investigated in terms of the vortex length and flow patterns for a wide range of flow rates. For this purpose, different parallel planes of the channel were investigated using flow visualization.

\subsection{Inertial effects}

In order to study the influence of Re on the vortex dynamics of Newtonian fluid flow, visualizations of the flow patterns near the expansion plane were performed for a wide range of flow rates $(R e<20)$ and various expansion ratios (ER $=2.4,4$ and 8). Here, the Reynolds number is based on upstream flow characteristics and defined as $R e=\rho U_{1}\left(2 H_{1}\right) / \eta$, where $U_{1}$ is the average velocity in the upstream channel. For the shear-thinning fluid, the viscosity is calculated using the sPTT model at a characteristic shear-rate $\left(\dot{\gamma}=U_{1} / H_{1}\right)$.

In Fig. 4 we compare the experimental and numerical flow patterns obtained at the center plane for all expansion ratios studied. The numerical predictions were carried out with the refined mesh (M80) in order to ensure high accuracy.

As can be observed in Fig. 4, a Moffatt vortex [14] is formed downstream of the expansion plane, for all expansion ratios. Increasing the flow inertia leads to an increase of the vortex size 

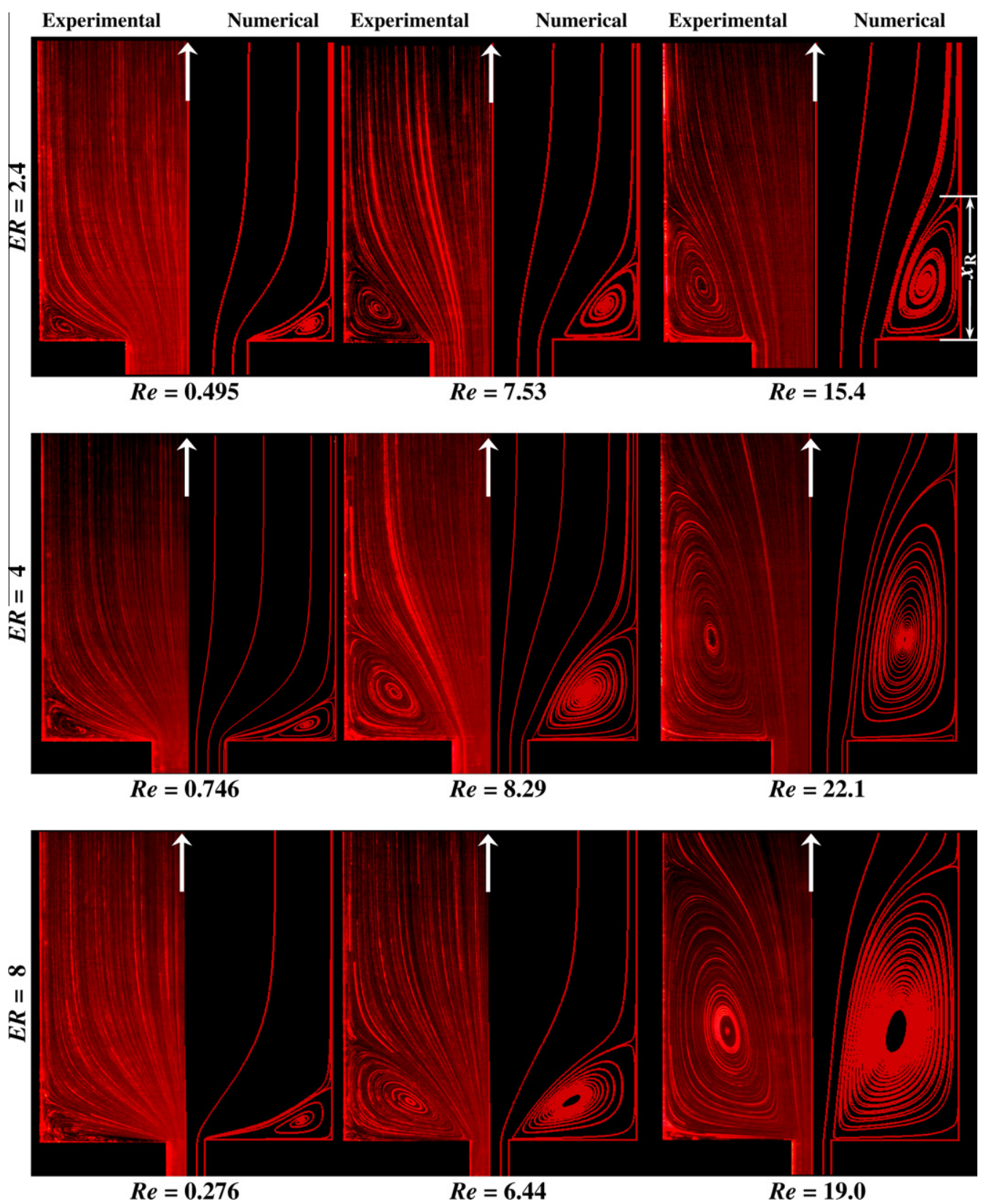

Fig. 4. Experimental and numerical flow patterns at the middle plane ( $y=0$ or $z=0$ ) for the Newtonian fluid at different Reynolds numbers and expansion ratios.

and an excellent agreement between the experimental results and numerical predictions is found. For Newtonian fluids, the formation and dynamics of the recirculations that appear downstream of an abrupt expansion is well-documented in the literature (e.g. $[17,18,24,25])$.

In Fig. 5 we analyze the effect of flow inertia on the measured normalized vortex length, $x_{\mathrm{R}} /\left(2 \mathrm{H}_{2}\right)$ (c.f. Fig. 4, $R e=15.4$ and $E R=2.4$ ) for the Newtonian fluid. Moreover, we also present the numerical predictions obtained with the three meshes (M40, M64 and M80) used for each expansion ratio.

For all expansion ratios studied, the vortex size increases monotonically when the Reynolds number is increased. For the whole range of Re studied, the numerical results obtained using meshes M40, M64 and M80 are in good agreement with the experimental results. Furthermore, the differences between the three meshes are small and the highest deviation between the results obtained is below $2 \%$, demonstrating the high accuracy of the numerical simulations.
For creeping flow conditions (i.e. in the limit when $R e \rightarrow 0$ ) the numerical simulations predict the following vortex dimensions: $x_{\mathrm{R}} /$ $\left(2 \mathrm{H}_{2}\right)=0.141$ for $\mathrm{ER}=2.4 ; \quad x_{\mathrm{R}} /\left(2 \mathrm{H}_{2}\right)=0.163$ for $\mathrm{ER}=4 ; \quad x_{\mathrm{R}} /$ $\left(2 \mathrm{H}_{2}\right)=0.174$ for $\mathrm{ER}=8$. These results are in agreement with the predictions for square-square contraction flows under creeping flow conditions (cf. $[8,43,44])$, a consequence of the reversibility of Newtonian inertialess flows, i.e. in the limit when $\operatorname{Re} \rightarrow 0$ the flow patterns for expansion and contractions flows are indistinguishable.

\subsection{Elastic effects}

In order to quantify the effect of viscoelasticity, we use the Deborah number, here defined based on upstream flow conditions, $D e=\lambda U_{1} / H_{1}$. Fig. 6 shows the flow patterns for both viscoelastic fluids obtained experimentally at the middle plane $(y=0$ or $z=0)$, for a range of Deborah numbers (or flow rates), covering the whole range of ER studied. 


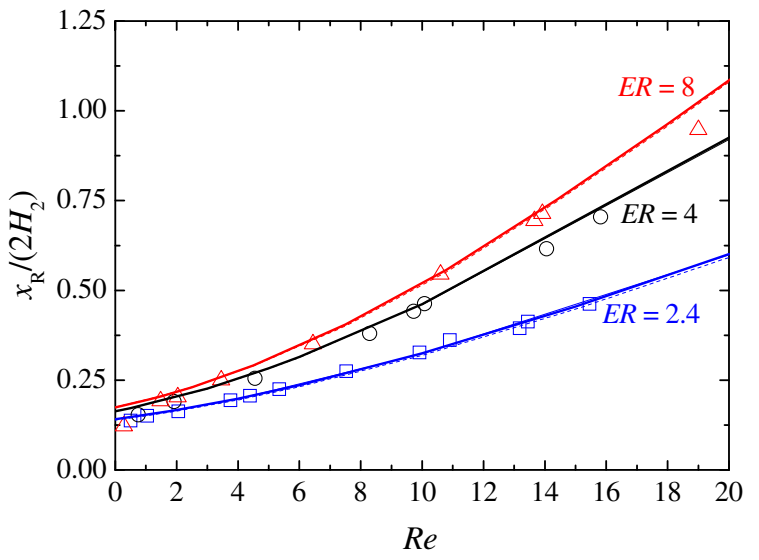

Fig. 5. Dimensionless vortex length for Newtonian fluid flow as a function of the Reynolds number for $\mathrm{ER}=2.4,4$ and 8 . The symbols represent the experimental data, the dashed lines represent the numerical predictions performed using mesh M40, the thin solid lines the numerical results obtained with mesh M64 and the thick solid lines the predictions using the more refined mesh, M80.

Comparing the flow patterns for both viscoelastic fluids, several similarities can be identified. For all expansion ratios studied, the flow of the two viscoelastic fluids presents a corner vortex downstream of the expansion plane and, in general, increasing the Deborah number leads to a decrease of the corner vortex length.

The dependence of the vortex length on the Deborah number, for all expansion ratios studied, is quantified in Fig. 7 for the Boger fluid and in Fig. 8 for the shear-thinning fluid. The vortex length is scaled with the side length of the downstream square duct $\left(2 \mathrm{H}_{2}\right)$. In addition, in Figs. 7 and 8 we also show the predictions obtained from the numerical simulations.

For the lower expansion ratios, ER = 2.4 and 4, the numerical predictions of the viscoelastic fluid flow were obtained using meshes M64 and M80 and there is a good agreement between the numerical results using both computational meshes, as shown in Figs. 7a and 8. For this reason, and since the computational time required for the runs with more refined meshes is substantially higher, for the simulations of the viscoelastic fluid flow through the $1: 8$ and $1: 12$ square-square expansions we used only meshes M64 or M60, respectively. Note that besides the high relaxation times used, particularly for the shear-thinning fluid, the increase of the number of cells for the refined meshes (e.g. for $E R=12$, the more refined mesh, M96, has five times more cells than mesh M60) renders the simulations of the viscoelastic fluid flow through 3D expansions with a high expansion ratio unfeasible in practice due to the very large CPU times involved (of the order of weeks for the highest $D e$ ).

At low Deborah numbers the numerical simulations predict accurately the creeping flow Newtonian plateaus for all fluids and expansion ratios $\left(x_{\mathrm{R}} /\left(2 \mathrm{H}_{2}\right)=0.141\right.$ for $\mathrm{ER}=2.4 ; x_{\mathrm{R}} /\left(2 \mathrm{H}_{2}\right)=$ 0.163 for $\mathrm{ER}=4 ; x_{\mathrm{R}} /\left(2 \mathrm{H}_{2}\right)=0.174$ for $\mathrm{ER}=8 ; x_{\mathrm{R}} /\left(2 \mathrm{H}_{2}\right)=0.177$ for $E R=12$ ), which are not evident from the experimental results (cf. Figs. 7a and 8). The comparison between the experimental results obtained with the Boger fluid and the numerical simulations using the Oldroyd-B model is displayed in Fig. 7a. The experimental data show a slight decrease of the vortex size with increasing $D e$, in contrast with the numerical simulations that display vortex suppression only up to $D e \approx 1.5$ and $D e \approx 6$ for $\mathrm{ER}=2.4$ and 12 ,

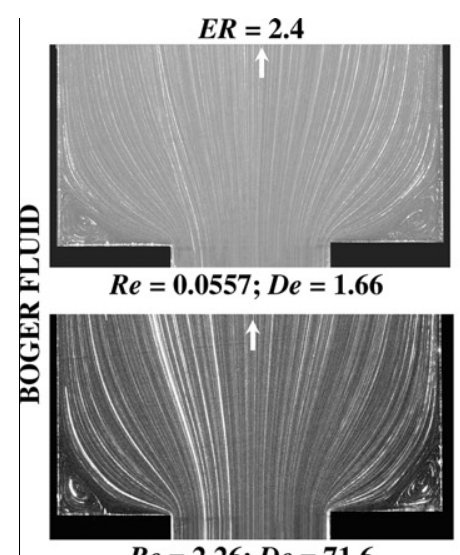

$R e=2.26 ; D e=71.6$

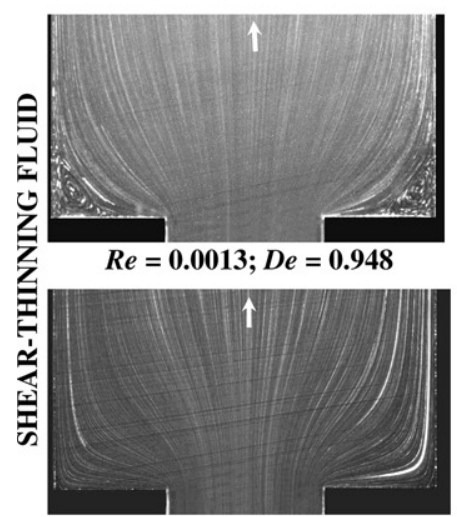

$R e=7.88 ; D e=368$

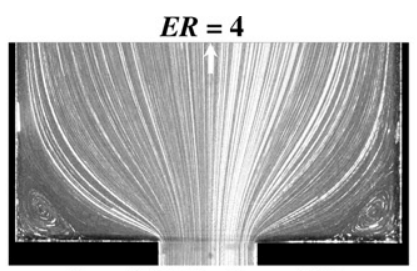

$R e=0.0571 ; D e=4.91$

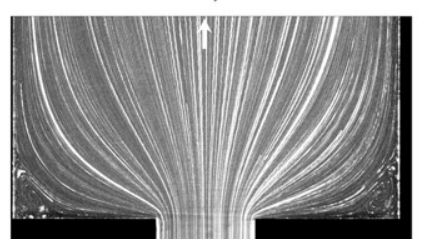

$R e=1.06 ; D e=74.9$

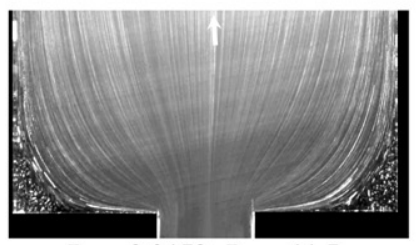

$R e=0.0152 ; D e=11.5$

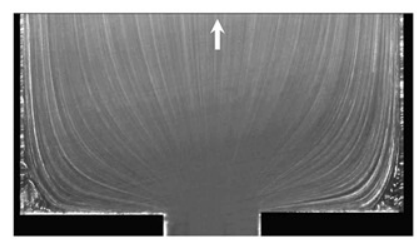

$R e=5.63 ; D e=570$

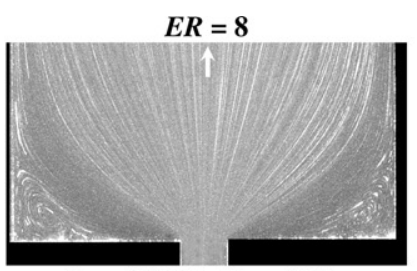

$R e=0.0295 ; D e=9.84$

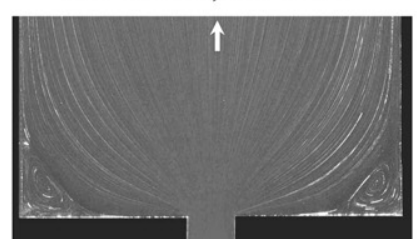

$R e=0.301 ; D e=104$

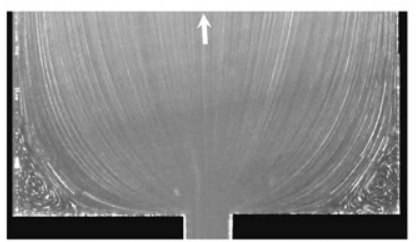

$R e=0.0518 ; D e=61.5$

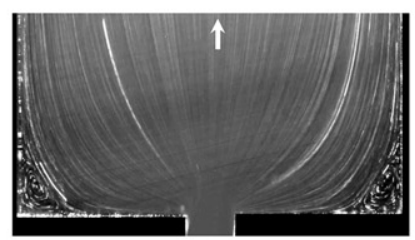

$R e=0.924 ; D e=439$

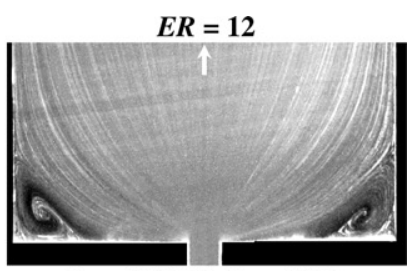

$R e=0.0176 ; D e=14.1$

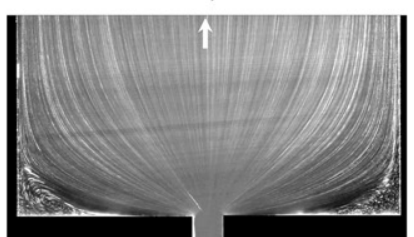

$R e=0.0695 ; D e=54.6$

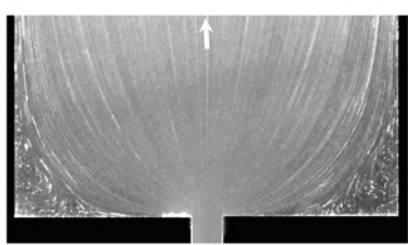

$R e=0.0724 ; \mathrm{D} e=135$

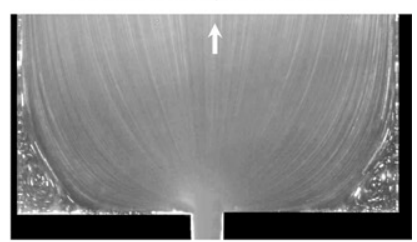

$R e=0.647 ; D e=603$

Fig. 6. Effect of elasticity on the Boger and shear-thinning fluid flow patterns at the middle plane for different expansion ratios. 

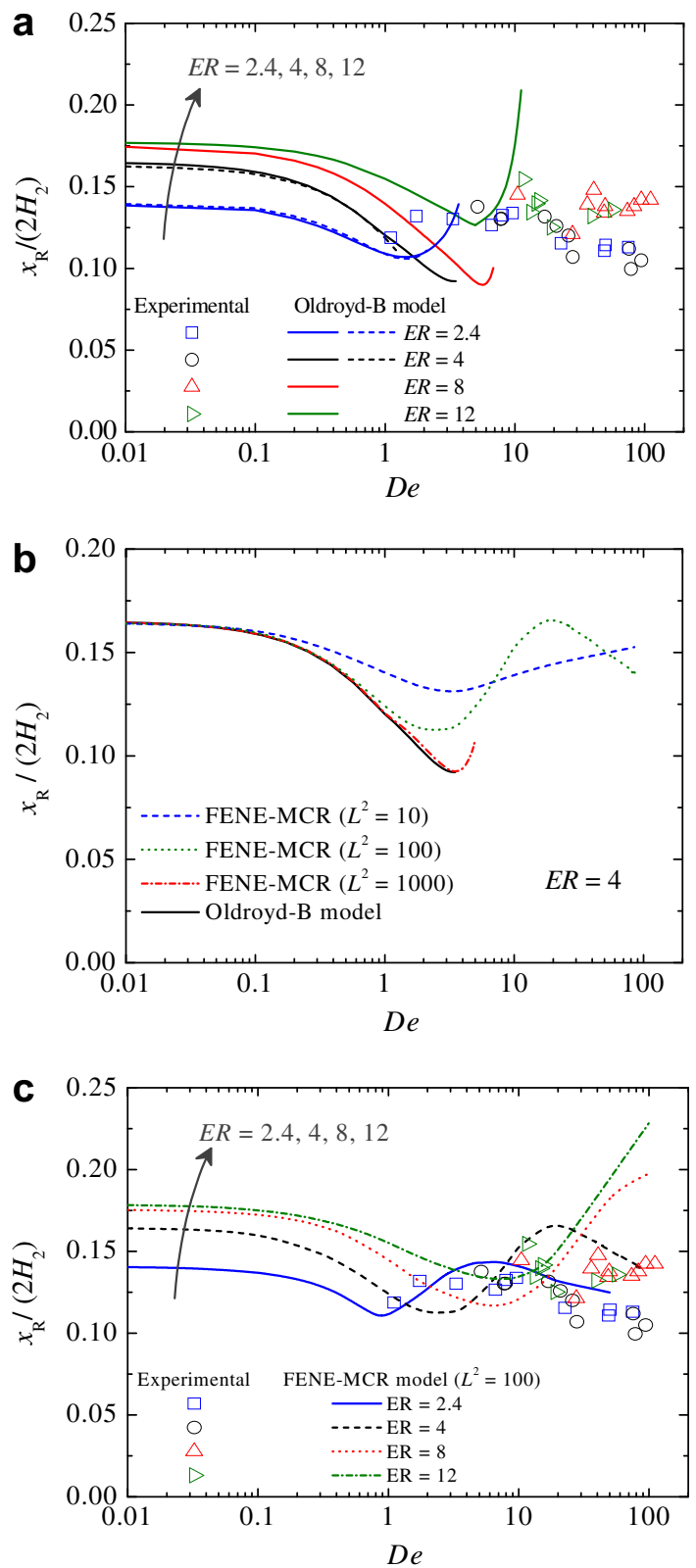

Fig. 7. Dimensionless vortex length as a function of the Deborah number for Boger fluid flow. (a) Comparison of experimental data (symbols) with predictions using the Oldroyd-B model for different ER (solid lines represent the numerical predictions using mesh M64 for $\mathrm{ER}=2.4,4$ and 8 or mesh $\mathrm{M} 60$ for $\mathrm{ER}=12$, and the dashed lines represent the numerical predictions using mesh M80 for ER $=2.4$ and 4); (b) Influence of parameter $L^{2}$ of FENE-MCR model for ER = 4 (mesh M64); (c) Comparison between FENE-MCR predictions $\left(L^{2}=100\right)$ and experimental results for different ER (meshes M60 for ER = 2.4, 4 and 8; mesh M64 for ER = 12).

respectively. At higher De significant vortex enhancement is predicted with the Oldroyd-B model, a phenomenon that is not reproduced in the experiments. Also, the convergence of the numerical simulations using the Oldroyd-B model is limited to a range of De significantly narrower than that achieved in the experiments. To enhance the numerical stability, we also performed numerical simulations using the FENE-MCR constitutive equation.

As detailed in Section 3, the values of parameters $\lambda, \eta_{\mathrm{P}}$ and $\eta_{\mathrm{S}}$ of the FENE-MCR model were the same as in the Oldroyd-B model. However, we were unable to select the appropriate value of $L^{2}$ based on steady or oscillatory shear data alone, and as such a base value of $L^{2}=100$ was chosen in agreement with previous studies

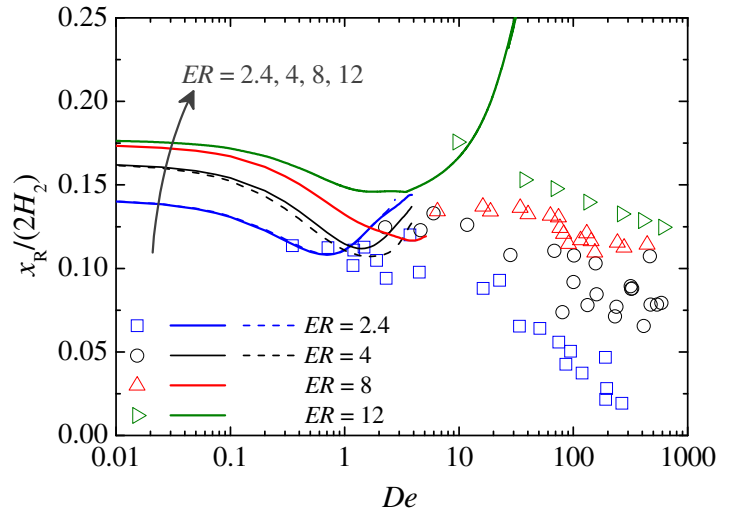

Fig. 8. Dimensionless vortex length as a function of the Deborah number for the shear-thinning fluid flow for all expansion ratios studied and comparison with numerical simulations using the sPTT model. The symbols represent experimental data, the solid lines represent the numerical predictions using mesh M64 for $\mathrm{ER}=2.4,4$ and 8 or mesh $\mathrm{M} 60$ for $\mathrm{ER}=12$ and the dashed lines represent the numerical predictions using mesh $\mathrm{M} 80$ for $\mathrm{ER}=2.4$ and 4 .

(e.g. [40]). To analyze the effect of $L^{2}$ on the numerical results, we show in Fig. 7b the vortex size predicted for $E R=4$ and a wide range of $L^{2}$ values (from a relatively low value of $L^{2}=10$ up to $L^{2}=1000$ ). For high $L^{2}$ (e.g. $L^{2}=1000$ ), the numerical results are similar to those obtained with the Oldroyd-B model, with very little improvement in terms of numerical stability and therefore in terms of the range of $D e$ we are able to achieve in the simulations. On the other hand, for low $L^{2}$ (e.g. $\left.L^{2}=10\right)$ the polymer molecules do not stretch significantly, and therefore elastic effects are suppressed, with a low influence of De on the vortex size. For intermediate $L^{2}$ values, we are able to observe a more pronounced influence of $D e$ on the flow characteristics. For $L^{2}=100$ an interesting behavior is observed in the predicted vortex size, with a minimum vortex size occurring at $D e \approx 2.5$, followed by a maximum value at $D e \approx 20$. This non-monotonic behavior is exclusively due to the elasticity of the fluid, since inertial effects are negligible, even at the larger De flow conditions. Indeed, for the higher flow rate cases we performed additional flow simulations assuming the limiting behavior of creeping flow (by dropping the convective term in the momentum equation) and the differences observed relative to the inertial case are negligible (in all cases below 1\%), confirming that inertia is not important here. We also note that the normal stresses are maximum along the upstream channel walls, and in the vicinity of the expansion plane corner (singularity), and the numerical simulations show an increase of $\operatorname{Tr}(\tau)$ when $L^{2}$ increases.

In Fig. 7c we compare the experimental measurements of vortex length for the various ER investigated with the predictions using the FENE-MCR model, assuming $L^{2}=100$, a value used hereafter in all the numerical simulations with the FENE-MCR model. The agreement between experiments and numerical simulations is now better than that found for the Oldroyd-B model, but still there are significant differences, indicating that more realistic constitutive equations are needed to better reproduce the behavior of the Boger fluid, probably using multimode models.

For the shear-thinning fluid, the decrease of the vortex size with $D e$ is more pronounced, in particular for the lower expansion ratios, as is visible in the experimental data shown in Fig. 8. The predictions using the sPTT model are restricted to a significantly narrower range of $D e$, as compared with the experiments, and the vortex reduction trend is only predicted at low De flow conditions. Interestingly, the same shear-thinning fluid was used in Ref. [41] to investigate the viscoelastic flow in square-square contractions [41], but in that case a much better agreement between 
the experiments and the numerical simulations (also using the sPTT model with the same parameters used in the present investigation, and the same viscoelastic flow solver) was observed with converged numerical simulations in a wider range of $D e$, showing that the expansion flow is a numerically more stringent flow problem.

\subsection{Three-dimensionality of the flow}

The previous investigations regarding the flow of Newtonian and viscoelastic fluids, through square-square contractions $[8,43,44]$, demonstrated that the flow in these geometries is highly three-dimensional. In order to study the 3D nature of the flow through square-square expansions, a detailed investigation at several parallel planes was carried out. Fig. 9 shows the visualized pathlines and the corresponding numerical predictions for the Newtonian fluid flow at different planes of the 1:8 square-square expansion, ranging from the center plane $\left(y / H_{2}=0\right.$ or $\left.z / H_{2}=0\right)$ to a plane near the wall of the channel $\left(y / H_{2}= \pm 0.875\right.$ or $z /$ $\mathrm{H}_{2}= \pm 0.875$ ). At the center plane the pathlines illustrated are real, while for the other planes the visualized flow shows the projections of the pathlines at the visualized plane. Again, we observe an excellent agreement between the visualizations and the numerical predictions.

For the range of flow rates studied, the flow is symmetric relative to the two center planes $(y=0$ and $z=0)$ and to the two diagonal planes $(y= \pm z)$. To further document the complex flow behavior in square-square expansions, in Fig. 10 we show a three-dimensional view of some streamlines, showing the open vortical structures predicted numerically with the refined mesh (M80) for the Newtonian fluid at creeping flow conditions ( $R e \rightarrow 0$ and $\mathrm{CR}=4$ ). To simulate creeping flow conditions we neglect the convective term in the momentum equation, but keep the transient term $(\rho \partial u / \partial t)$ and use a pseudo-time marching algorithm to achieve steady flow conditions. When steady-state is achieved, the transient term vanishes, and we obtain exactly creeping flow conditions $(R e=0)$.

Under creeping flow, the streamlines generated by the Newtonian fluid flowing through a square-square expansion are coincident with those documented previously for the flow through square-square contractions $[8,43,44]$ due to the reversibility of inertialess flows. However, in the present configuration the flow occurs in the opposite direction, causing the fluid that is flowing near the center plane wall of the upstream channel to pass through the expansion plane and enter the recirculation in the center plane (plane EFGH in Fig. 10c). Once there, the fluid rotates around the center of the recirculation and follows a helical trajectory toward the diagonal plane $(A B C D)$, where it turns now to the periphery and exits the recirculation moving toward the exit of the downstream channel close to the diagonal plane wall, as illustrated in Fig. 10c. The streamlines in the center and diagonal planes are shown in Fig. 10a and b, respectively, to better illustrate the dynamics of the secondary flow in the symmetry planes.
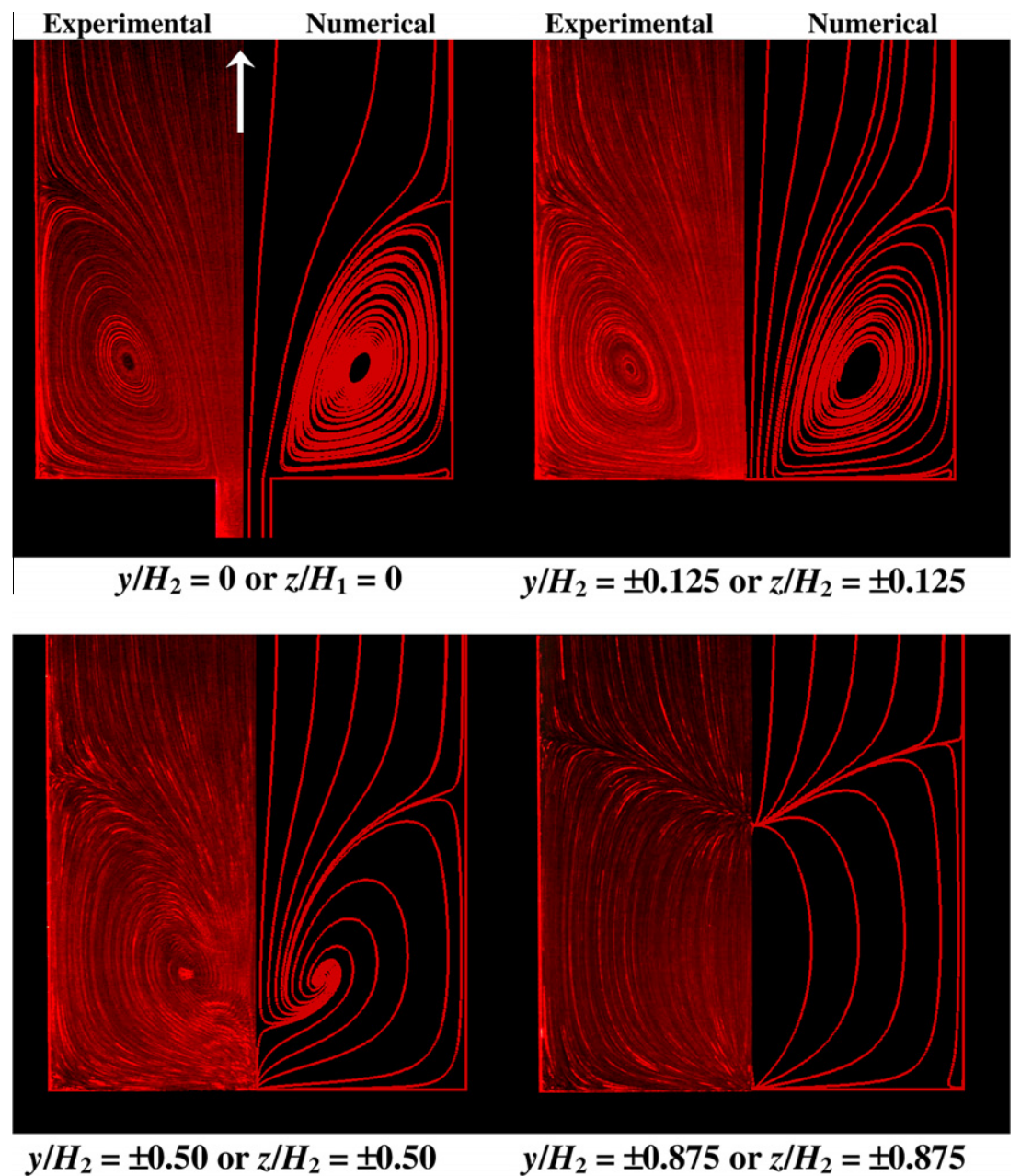

Fig. 9. Experimental and numerical projected pathlines of the Newtonian fluid flow at different planes of the 1:8 square-square expansion for Re =13.9. 
a

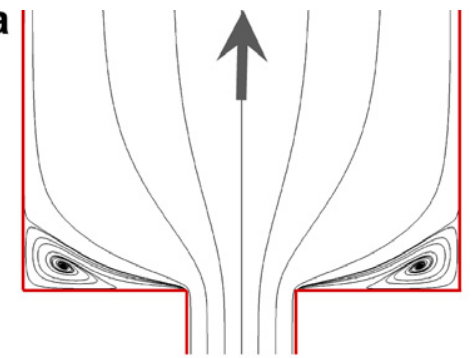

b

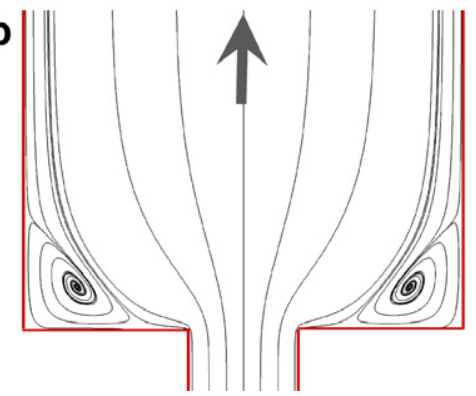

C

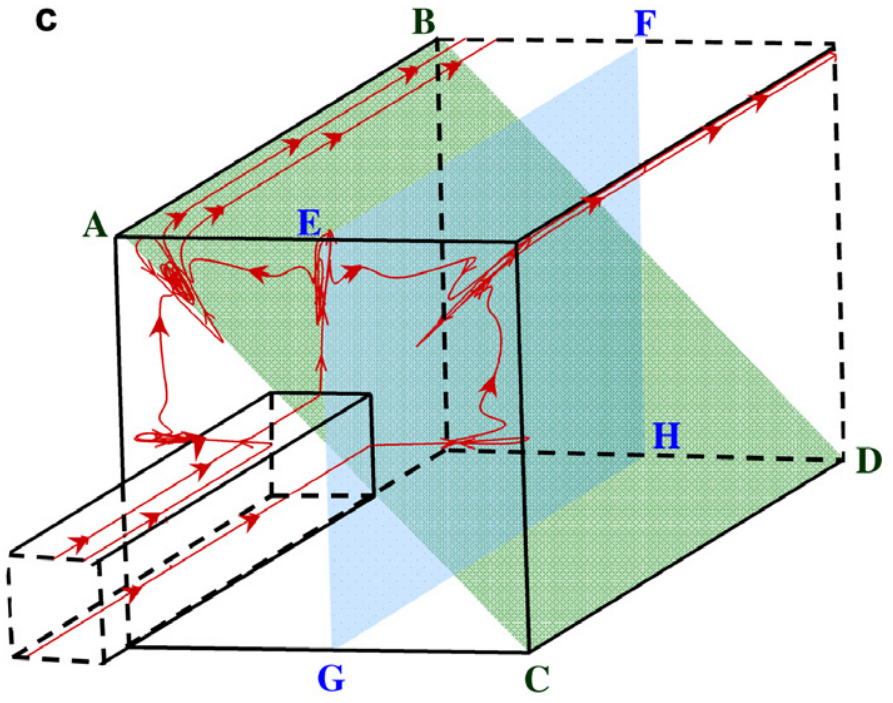

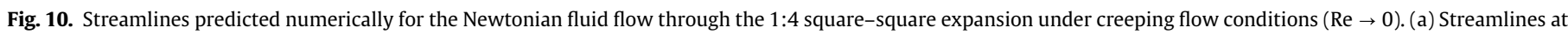

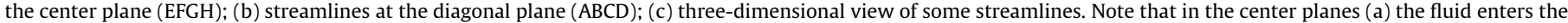
recirculation while in the diagonal planes (b) the fluid exits the recirculation.

Interestingly, when inertial effects are important, besides the increase of the recirculation documented previously (cf. Figs. 4 and 5) we observe a reversal in the flow direction within the recirculation. This can be observed in the streamlines plotted in Fig. 11 at $R e=10$ and $\mathrm{CR}=4$. In this case, the recirculating flow occurs from the diagonal planes, where the fluid is sucked in (cf. Fig. 11b), to the center planes where the fluid is ejected (cf. Fig. 11a). Such a flow reversal was previously documented in square-square contractions, but was attributed to elastic effects $[8,43,44]$. The present results suggest that flow reversal in square-square contractions or expansions is not a fingerprint of elastic effects, but seems to occur concomitantly with vortex enhancement, which is inertially-driven in expansion flows and elasticity-driven in contraction flows.

In Fig. 12 we show pathline projections of the Boger and shear-thinning fluid flows at different parallel planes of the 1:2.4
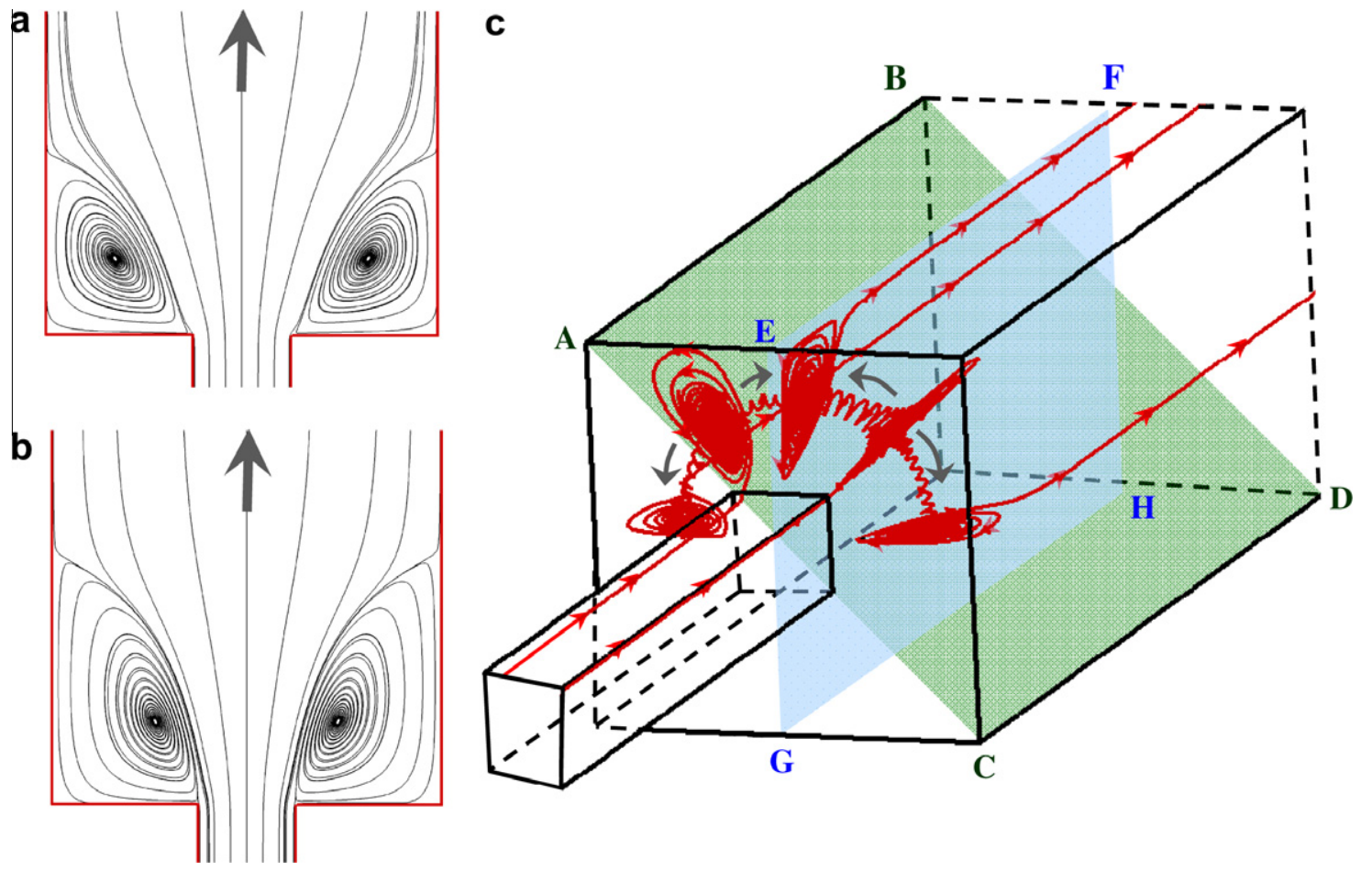

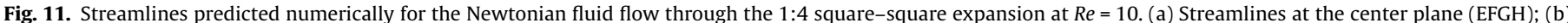

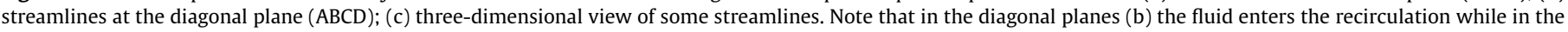
central planes (a) the fluid exits the recirculation. 

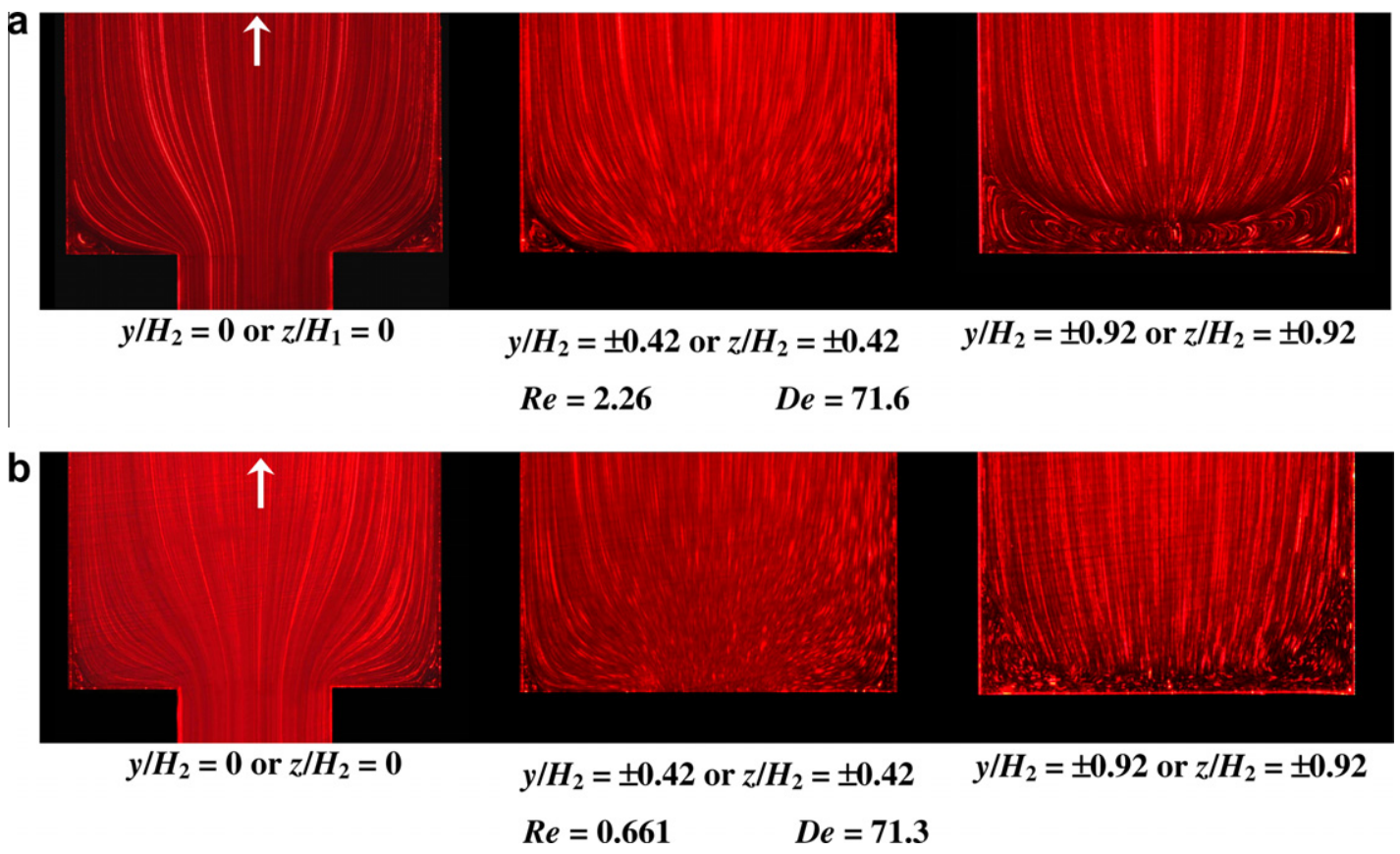

Fig. 12. Projections of pathlines at different parallel planes for the Boger (a) and shear-thinning (b) fluid flow through the square-square expansion (ER = 2.4).

square-square expansion and identical Deborah numbers, illustrating the complex and highly three-dimensional flow behavior particularly near the walls.

\section{Velocity field}

\subsection{Newtonian fluid}

In order to highlight the inertial effects on the velocity field, in Fig. 13 we show axial velocity profiles along the centerline for two expansion ratios ( $E R=2.4$ and $E R=8$ ), at different Reynolds numbers. The axial velocity profile predicted numerically for negligible inertial flow conditions (creeping flow) is also shown for comparison purposes. Moreover, we compare the experimental results with numerical predictions for each value of $R e$.

In all cases, the dimensionless velocity profiles are plotted from locations upstream $(x<0)$ to downstream $(x>0)$ of the expansion plane. As can be seen, the dimensionless velocity gradient downstream of the expansion plane decreases when the Reynolds number increases. As inertia increases, entrance effects also become more pronounced and the size of the recirculations, formed downstream of the expansion plane, also increases. For ER $=2.4$, the normalized velocity profile measured experimentally at low $R e$ is similar to that obtained numerically for creeping flow. For all axial velocity profiles shown in Fig. 13, the experimental results are in good agreement with those predicted numerically, for both $\mathrm{ER}=2.4$ and 8 , thus validating the PIV measurements.

To further attest the good agreement between experimental and numerical results, in Fig. 14a we present a comparison between the middle plane pathlines obtained using three different approaches: flow visualizations using streak photography; integration of the measured velocity field using PIV; numerical predictions. The example shown corresponds to Newtonian fluid flow through the 1:4 square-square expansion at similar Reynolds numbers $(R e \approx 10)$. In Fig. $14 \mathrm{~b}$ we compare the normalized velocity magnitude contour plots obtained from the PIV measurements and from the numerical simulations. Again, the experimental results are in excellent agreement with the numerical predictions.
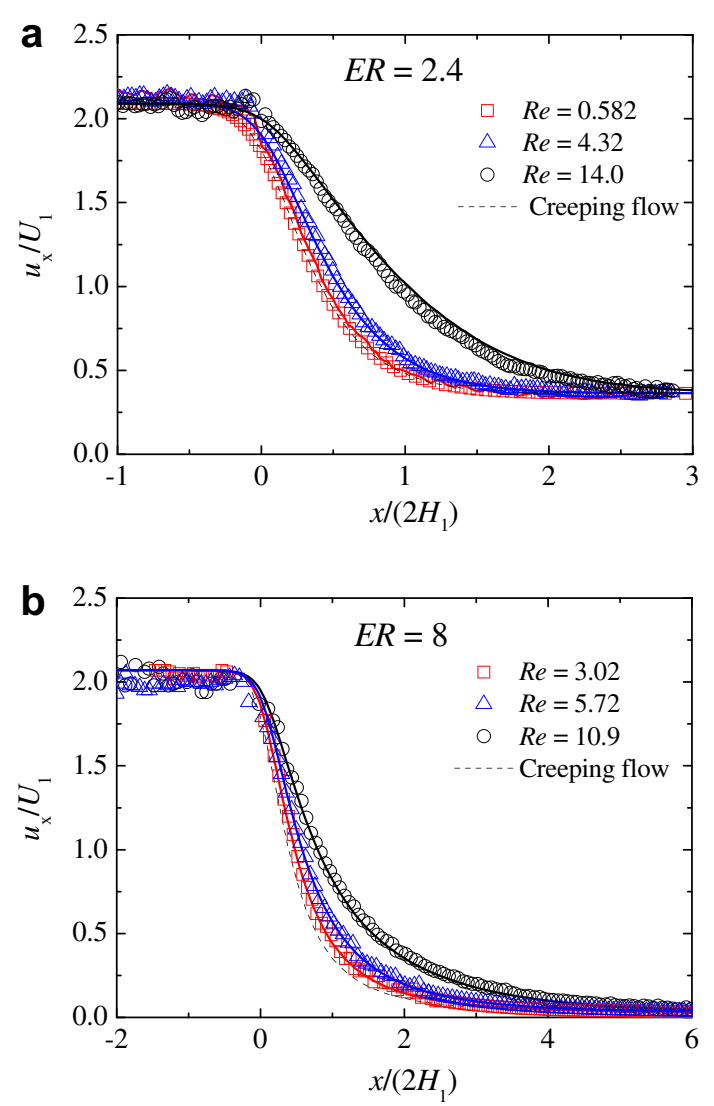

Fig. 13. Experimental (symbols) and numerical (lines) axial velocity profiles at the centerline for the Newtonian fluid flow at different $R e$ : (a) $E R=2.4$; (b) $E R=8$.

\subsection{Viscoelastic fluid}

Fig. 15 shows normalized axial velocity profiles taken along the centerline for the Boger fluid flowing through the 1:4 and 1:12 
a

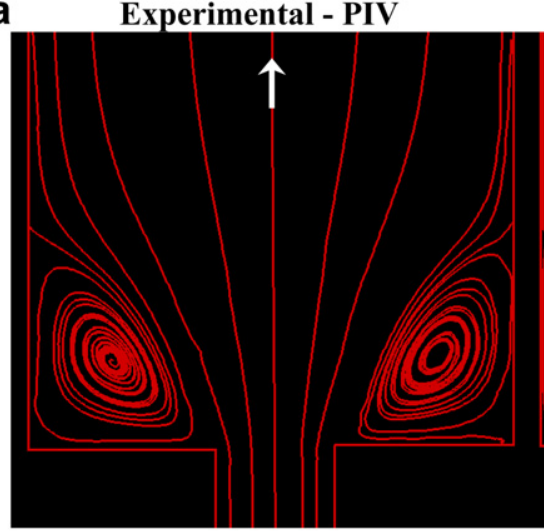

$R e=9.64$

b

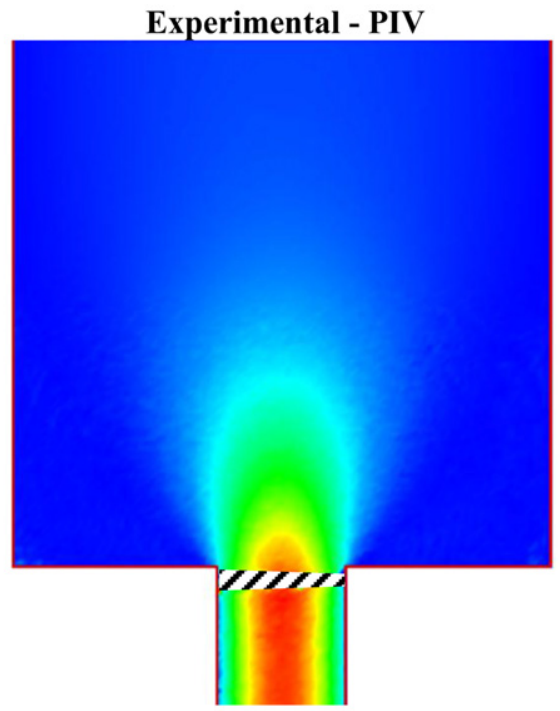

$R e=9.64$
Numerical

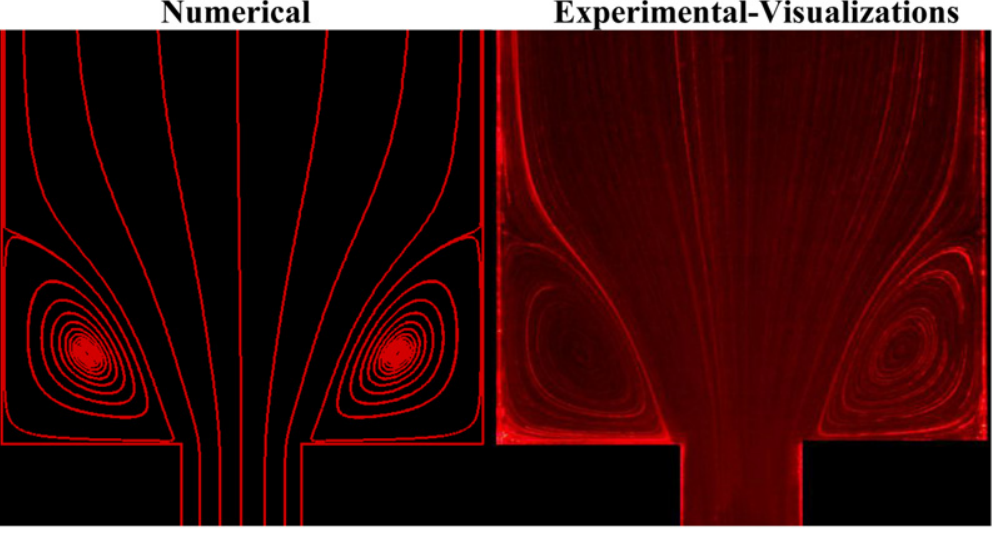

$\operatorname{Re}=\mathbf{9 . 6 4}$

$R e=9.73$

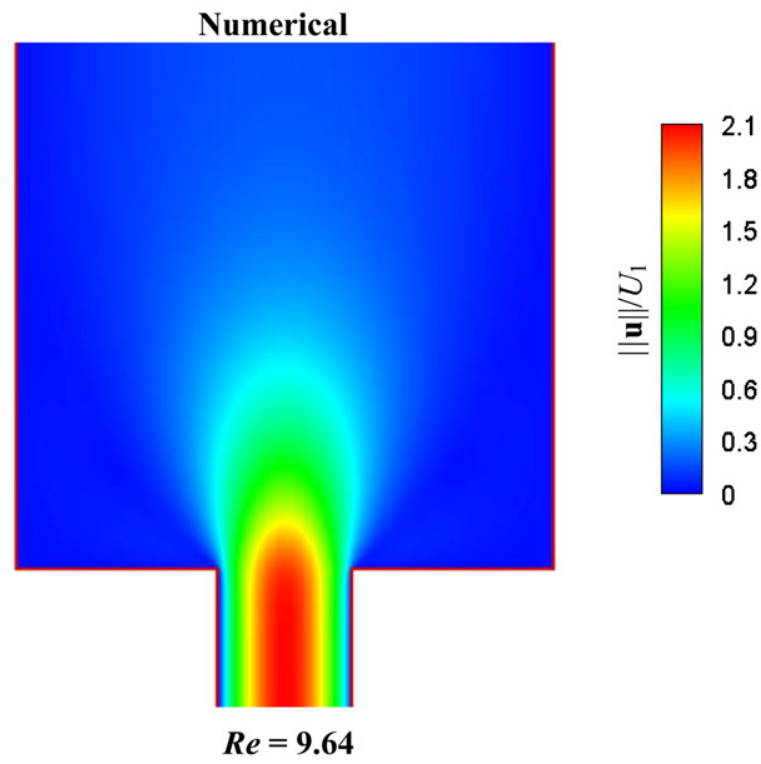

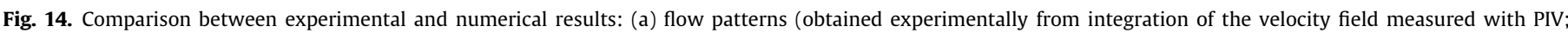
obtained from numerical calculations; obtained using long time exposure streak line photography); (b) normalized velocity magnitude contour plots.

square expansions (Fig. 15a) and for the shear-thinning fluid flowing through square-square expansions with expansion ratios of 2.4 and 8 (Fig. 15b). Predictions of dimensionless velocity profiles for a Newtonian fluid flowing at creeping flow conditions are also shown in Fig. 15 to highlight the influence of elasticity on the dimensionless velocity profiles.

For a square channel, the theoretical maximum velocity or centerline velocity is 2.096 times the average velocity for a constant viscosity fluid (either Newtonian or a Boger fluid) [44]. Therefore, for the Boger fluid, the axial velocity far upstream of the expansion should be $u_{x} / U_{1}=2.096$ as observed for the computed profiles for Newtonian and FENE-MCR fluids. In the limit $D e \rightarrow 0$, obtained experimentally for very low flow rates, the axial dimensionless velocity profile approaches that of a Newtonian fluid under creeping flow conditions. An increase in the flow rate (or $D e$ ) leads to the appearance of a local maximum of the axial velocity near the expansion region, which is also predicted numerically using the FENE-MCR model, although with some differences observed due to the inability of this model to accurately reproduce the complex behavior of the Boger fluid. For the Boger and the shearthinning fluids studied, at high $D e$, a significant overshoot on the axial velocity along the centerline is clearly visible with the maximum axial velocity reaching values well above the fully-developed value, which means that the fluid experiences an acceleration as it is approaching the expansion plane (converging streamlines) leading to a higher rate of decay of the velocity when it enters the channel with larger cross-section. The same phenomenon was observed experimentally by Rothstein and McKinley [45] for Boger fluid flow through an axisymmetric contraction-expansion and numerically by Oliveira [29] for a FENE-CR fluid and by Poole et al. [23] for UCM and sPTT fluids flowing through planar expansions, although to a smaller extent. Eventually, the centerline velocity downstream of the expansion reaches the theoretical value corresponding to fully-developed flow conditions and therefore good agreement between experiments and numerical simulations is observed. However, as the flow rate is increased, fully-developed flow is only achieved progressively farther away from the expansion plane and the overshoot on the velocity profile exhibits a higher magnitude, particularly for the shear-thinning fluid.

For the shear-thinning fluid, the ratio of the maximum velocity achieved in the upstream channel to the average velocity, depends on the flow rate, since the viscosity depends strongly on the shear rate. Thus, in order to predict the value of the axial velocity for fully-developed conditions, which was not measurable experimentally, we performed numerical simulations using a generalized Newtonian fluid (GNF) with a rheological behavior in steady shear 


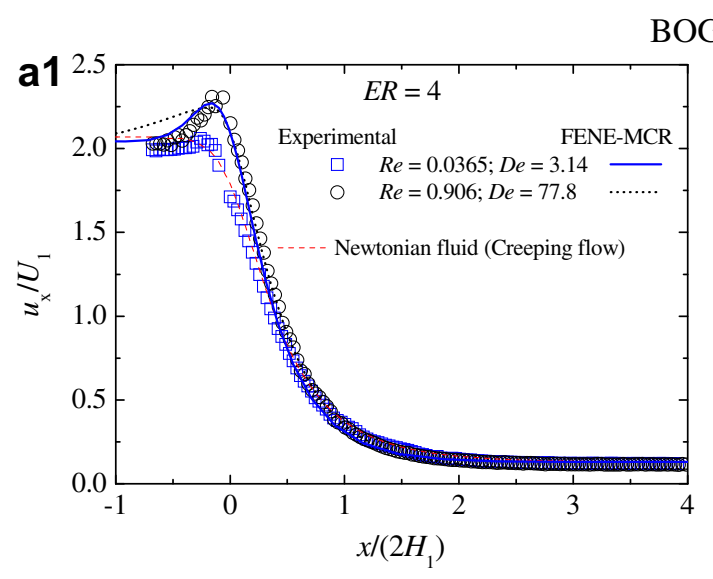

BOGER FLUID
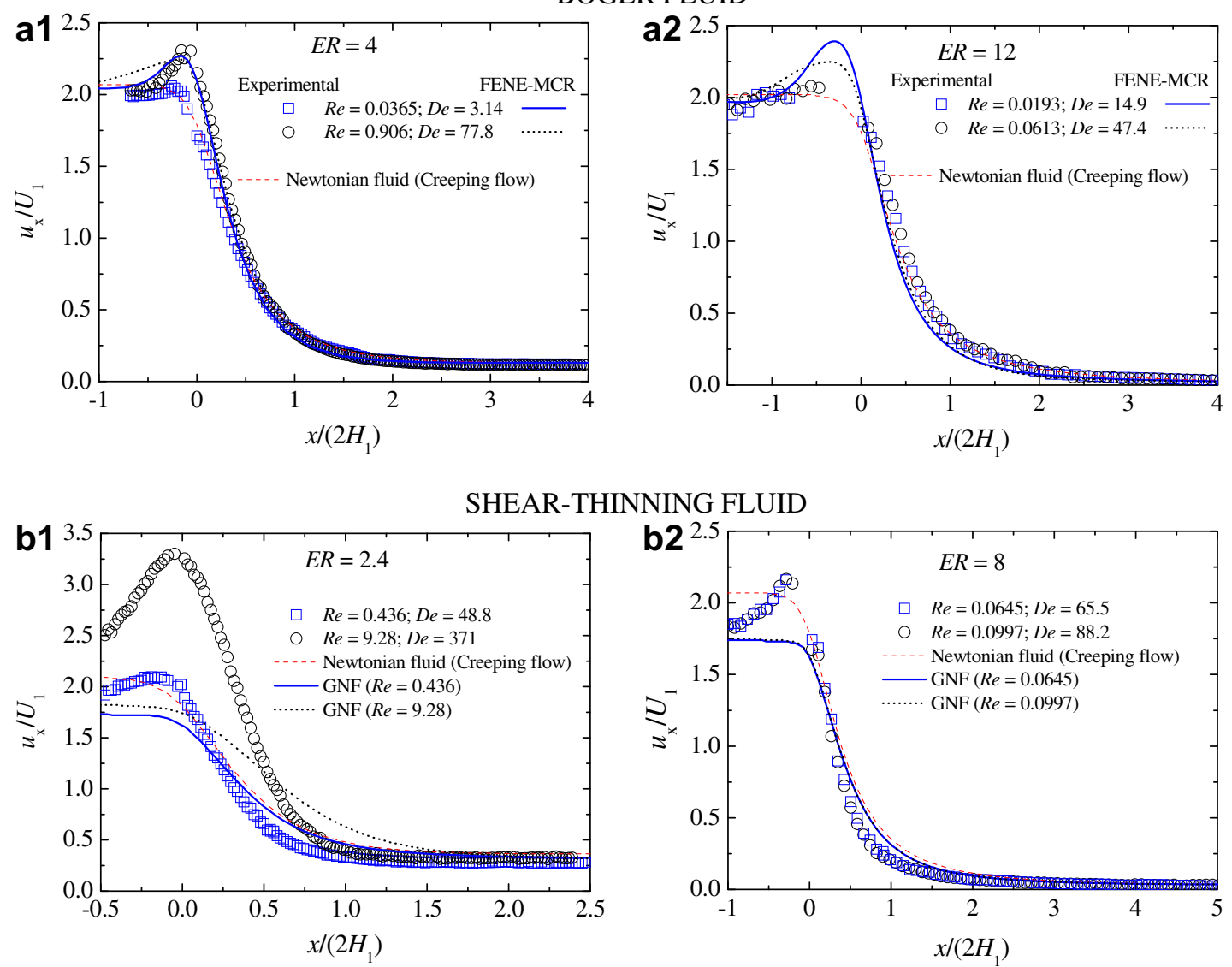

SHEAR-THINNING FLUID

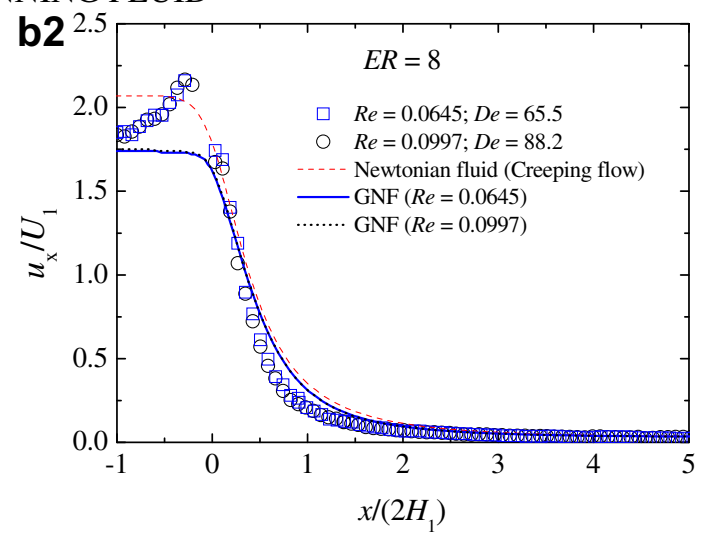

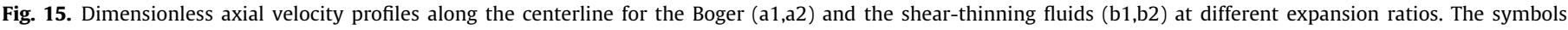
represent the experimental results and the lines represent the numerical predictions.

flow similar to that found for the shear-thinning viscoelastic fluid used. For this purpose, the rheological data (cf. Section 2.3) was fitted using a Carreau-Yasuda model [46],

$\eta=\eta_{\mathrm{S}}+\frac{\eta_{0}-\eta_{\mathrm{S}}}{\left[1+(\Lambda \dot{\gamma})^{a}\right]^{(1-n) / a}}$

where the parameters of the fitted model are: $\eta_{0}=1.65 \mathrm{~Pa}$, $\eta_{\mathrm{S}}=0.03$ Pa s, $\Lambda=10 \mathrm{~s}, n=0.36$ and $a=0.9$. The numerical predictions using this rheological model are also shown in Fig. 15b for the shear-thinning fluid. As can be seen, the fully developed normalized axial velocity depends on the flow rate and its value is smaller than that for constant viscosity fluids. Moreover it is possible to further attest that the overshoot present on the axial velocity profile is a consequence of the elastic effects, since for a GNF no overshoot is predicted.

In Fig. 16 we present the profiles of the $x$ - and $y$ - velocity components taken in the spanwise direction at different $x$-locations. Additionally, we also compare in Fig. 16(a1) the measured velocity profiles with the predictions using the FENE-MCR model with $L^{2}=100$ (for the Boger fluid) and in Fig. 16(b1) with the GNF fluid simulations (for the shear-thinning fluid). The predictions of the FENE-MCR model are in good agreement with the experimental measurements for the axial locations illustrated in Fig. 16a. However, in the vicinity of $x /(2 H 1) \approx-0.5$ the predicted centerline velocity is slightly higher than the experiments (cf. Fig. 15a1).

From the velocity profiles taken at discrete $x$-positions it is possible to infer about the evolution of the velocity along the channel. For the Boger fluid (cf. Fig. 16a), the velocity profile at location
$x=-H_{1}$ approaches fully-developed flow conditions, since the transverse velocity component is negligible and the maximum axial velocity on the centerline approaches the theoretical value $u_{x} / U_{1}=2.096$, valid for fully developed flow conditions of a constant viscosity fluid. In the channel with a larger cross-section, the $x$-component of the velocity decreases progressively while the $y$-component experiences an increase near its entrance and up to $x \approx 0.5 H_{1}$, gradually decreasing for locations farther downstream. For the shear-thinning fluid, the flow behavior is similar to that observed for the Boger fluid, except for the shape of the velocity profile. In this case, the profile becomes more like a plug flow profile, especially upstream of the expansion, a typical behavior of shear-thinning fluids under fully-developed flow conditions. Good agreement with the fully developed flow velocity profile predicted with the GNF fluid is observed for the shear-thinning fluid, as shown in Fig. 16 (b1) at the upstream location $\left(x=-H_{1}\right)$. However, closer to the expansion plane, the comparison between the experimental stream wise velocity profile and the GNF calculations become less accurate, due to the influence of elastic effects which are not captured in the GNF simulations.

\subsection{Pressure drop}

Pressure drop measurements for viscoelastic fluid flows through expansions are scarce, particularly if the flow occurs in channels with a 3D geometrical arrangement. The results obtained in this study for the Boger fluid flow through square-square expansions with different ER can be useful as benchmark data for validation of numerical results. 
BOGER FLUID

$(R e=0.906$ and $D e=77.8)$
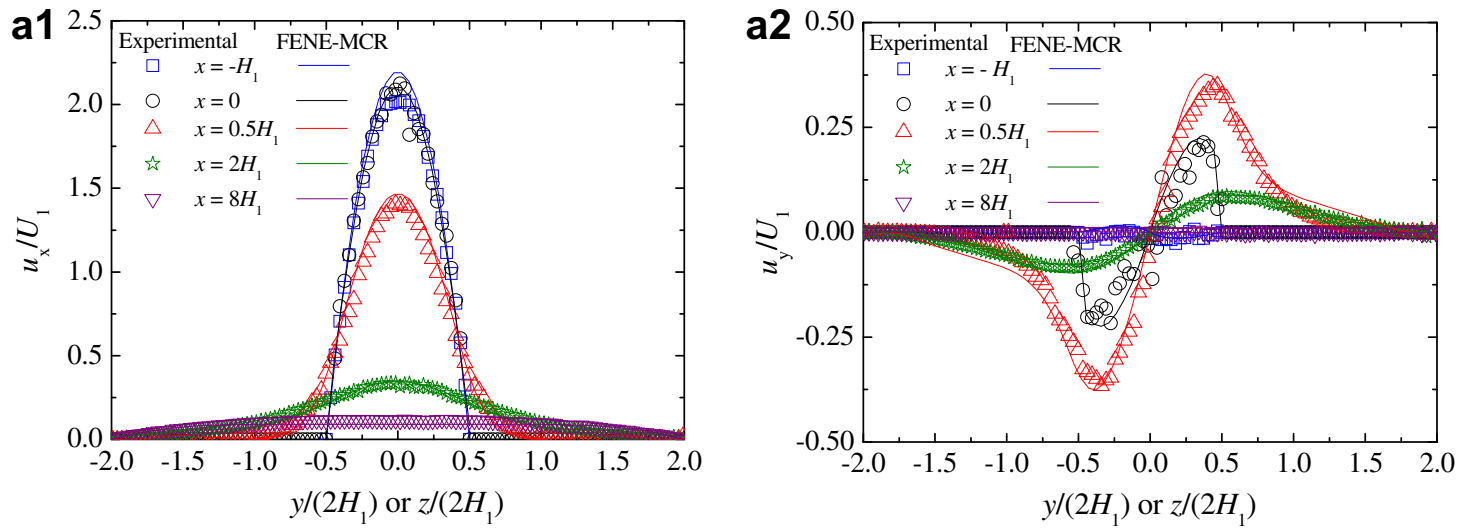

SHEAR-THINNING FLUID

$(R e=0.372$ and $D e=83.2)$
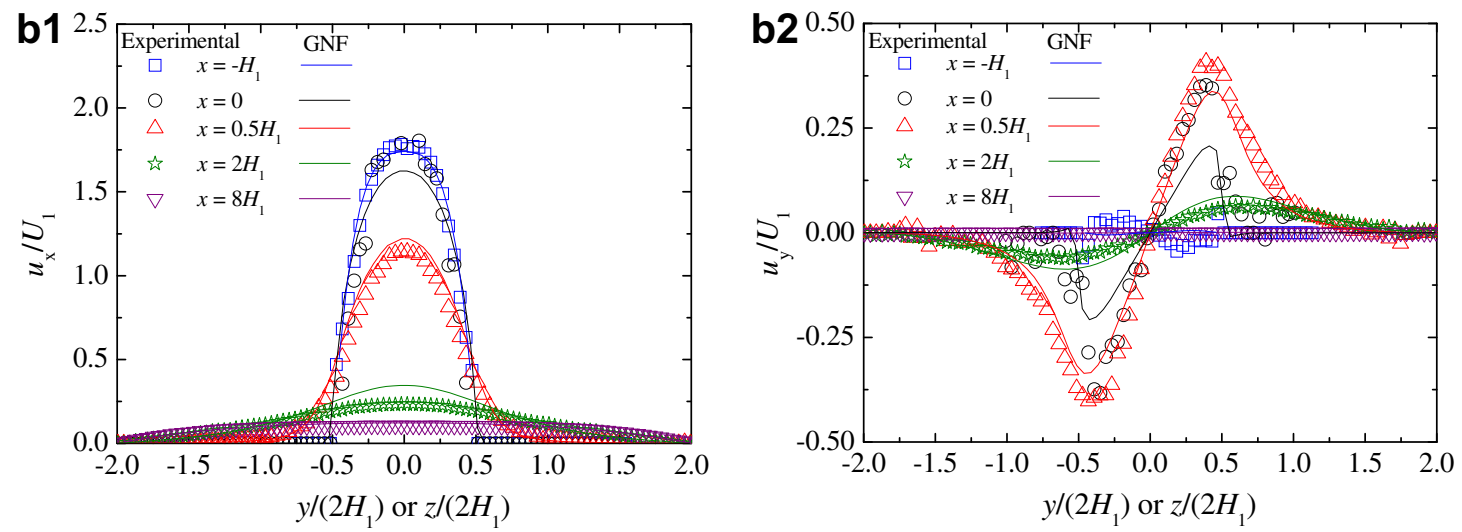

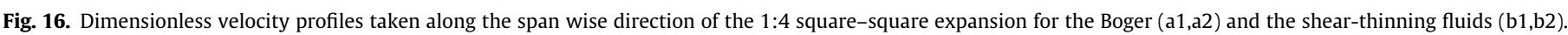

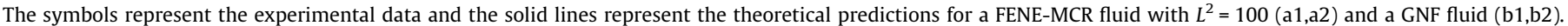

Fig. 17 shows the pressure drop measured with the Boger fluid (c.f. Section 2.2 for pressure ports locations) as a function of the flow rate for $E R=4,8$ and 12. Since the pressure drop increases linearly with the flow rate for all ER studied, we also present a linear fit to the experimental results. The predictions using the FENEMCR model are also included, and show a larger pressure drop than the experimental measurements. This observation is not totally unexpected since the FENE-MCR model predicts a constant shear viscosity, while the Boger fluid used in the experimental work exhibits some shear-thinning, although to a small degree. Ideally a Boger fluid should have a constant shear viscosity, but in practice real fluids exhibit some degree of shear thinning, which should be minimized as much as possible. For the Boger fluid used in the experiments, the average shear viscosity measured in steady shear flow for the range of shear rates where accurate measurements are obtained is approximately $0.45 \mathrm{~Pa} \mathrm{~s}$, about $30 \%$ below the shear viscosity of the FENE-MCR fluid used in the numerical simulations, which was based on a 3-mode plus solvent fit to small oscillatory shear data [8]. It is therefore not surprising that the predictions of the FENE-MCR model are on average about $20 \%$ higher than the experimental measurements. In Fig. 17 we also present the pressure drop predicted for a Newtonian fluid with a shear viscosity equal to the total shear viscosity of the FENE-MCR model used to fit the rheology of the Boger fluid ( $\eta_{0}=0.646 \mathrm{~Pa}$ s) and a Newtonian fluid with a shear viscosity equal to the solvent contribution of the shear viscosity of the FENE-MCR model $\left(\eta_{\mathrm{S}}=0.367 \mathrm{~Pa} \mathrm{~s}\right)$. As illustrated in Fig. 17, the relation between experimental and numerical results is analogous for all expansion ratios studied and the experimental data lies in-between the numerical predictions of the Newtonian fluids. From these results we can also conclude that there is no significant enhancement of pressure drop due to elastic effects, since the difference between the FENE-MCR predictions and the Newtonian fluid with a similar shear viscosity $(\eta=0.646 \mathrm{~Pa} \mathrm{~s})$ is small, in contrast with the results obtained with the same fluid in 3D square-square contractions where a significant increase of the entry pressure drop due to elastic effects was found for high contraction ratios [8].

\section{Conclusions}

The three-dimensional flow of a Newtonian and two viscoelastic fluids through square/square expansions with expansion ratios of $2.4,4,8$ and 12 was investigated experimental and numerically. In addition to the characterization of the flow through squaresquare expansions, this work also intends to provide useful data for benchmarking in a complex 3D flow.

Three-dimensional numerical simulations of the Newtonian and non-Newtonian fluid flow were performed using a finite volume method. The Newtonian fluid flow presents a Moffatt corner vortex downstream of the expansion plane and the effect of inertia on the flow behavior is similar for all expansion ratios studied: increasing the Reynolds number leads to an increase of the vortex length and intensity and a reversal in the flow direction inside the 

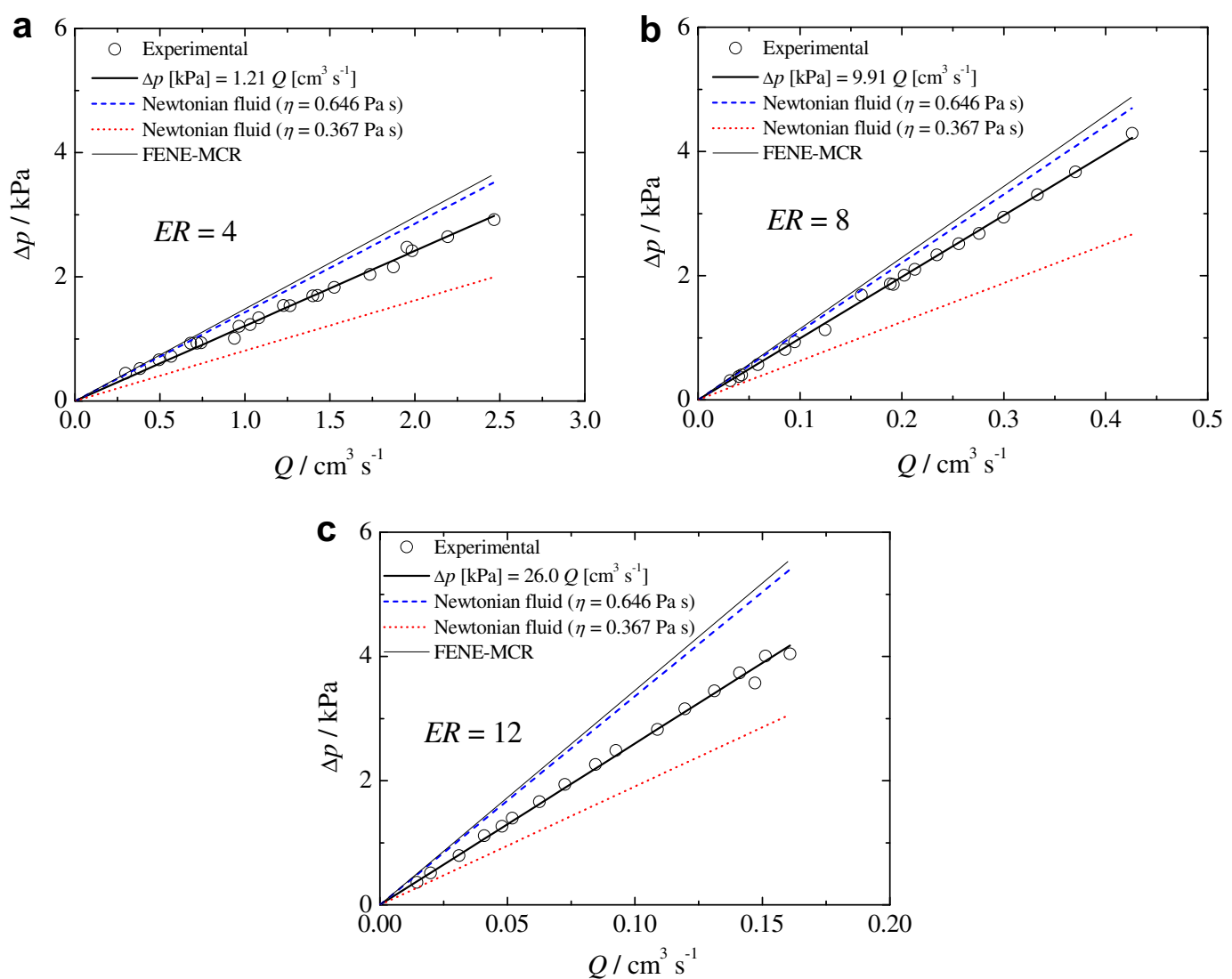

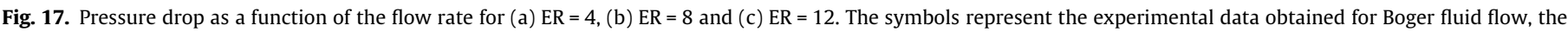

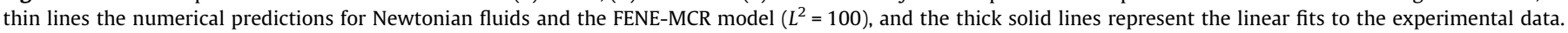

recirculation. The viscoelastic fluid flow behavior is also analogous for all expansion ratios studied: a corner vortex is observed downstream of the expansion plane and increasing the Deborah number leads, in general, to a decrease of the corner vortex length, which is more marked for the shear-thinning fluid. A complex helicoidal flow within the vortical structure is observed experimentally for all fluids studied and confirmed by the numerical simulations. The numerical results capture very well the flow characteristics obtained experimentally for the whole range of conditions for the Newtonian fluid. For the viscoelastic fluid flow the numerical simulations predict a decrease of vortex activity at low Deborah number flows, followed by a vortex enhancement at higher $D e$, a phenomenon not observed in the experiments. For the Boger fluid, the pressure drop across the square-square expansion increases linearly with the flow rate and does not reveal an enhancement of the extra pressure drop due to elasticity.

\section{Acknowledgements}

The authors acknowledge the financial support provided by Fundação para a Ciência e a Tecnologia (FCT) and FEDER through projects PTDC/EME-MFE/70186/2006, PTDC/EQU-FTT/71800/ 2006, REEQ/262/EME/2005 and REEQ/928/EME/2005. P.C. Sousa also acknowledges FCT for financial support through scholarship SFRH/BD/28846/2006.

\section{References}

[1] D.V. Boger, Viscoelastic flows through contractions, Ann. Rev. Fluid Mech. 19 (1987) 157-182.
[2] J.P. Rothstein, G.H. McKinley, Extensional flow of a polystyrene Boger fluid through a 4:1:4 axisymmetric contraction/expansion, J. Non-Newt. Fluid Mech. 86 (1999) 61-88.

[3] G. Mompean, M. Deville, Corrigendum to "Unsteady finite volume simulation of Oldroyd-B fluid through a three-dimensional planar contraction", J. NonNewt. Fluid Mech. 103 (2002) 271-272. J. Non-Newt. Fluid Mech. 72 (1997) 253-279.

[4] S. Nigen, K. Walters, Viscoelastic contraction flows: comparison of axisymmetric and planar configurations, J. Non-Newt. Fluid Mech. 102 (2002) 343-359.

[5] M.A. Alves, P.J. Oliveira, F.T. Pinho, On the effect of contraction ratio in viscoelastic flow through abrupt contractions, J. Non-Newt. Fluid Mech. 122 (2004) 117-130.

6] L.E. Rodd, T.P. Scott, D.V. Boger, J.J. Cooper-White, G.H. McKinley, The inertioelastic planar entry flow of low-viscosity elastic fluids in micro-fabricated geometries, J. Non-Newt. Fluid Mech. 129 (2005) 1-22.

[7] M.S.N. Oliveira, P.J. Oliveira, F.T. Pinho, M.A. Alves, Effect of contraction ratio upon viscoelastic flow in contractions: the axisymmetric case, J. Non-Newt. Fluid Mech. 147 (2007) 92-108.

[8] P.C. Sousa, P.M. Coelho, M.S.N. Oliveira, M.A. Alves, Three-dimensional flow of a Newtonian and Boger fluids in square-square contractions, J. Non-Newt. Fluid Mech. 160 (2009) 122-139.

[9] F.N. Cogswell, Converging flow and stretching flow: a compilation, J. NonNewt. Fluid Mech. 4 (1978) 23-38.

[10] D.M. Binding, An approximate analysis for contraction and converging flows, J. Non-Newt. Fluid Mech. 27 (1988) 173-189.

[11] T.-K. Hung, E.O. Macagno, Laminar eddies in a two-dimensional conduit expansion, La Houille Blanche 21 (1966) 391-401.

[12] E.O. Macagno, T.-K. Hung, Computational and experimental study of a captive annular eddy, J. Fluid Mech. 28 (1967) 43-64.

[13] F. Durst, A. Melling, J.H. Whitelaw, Low Reynolds number flow over a plane symmetric sudden expansion, J. Fluid Mech. 64 (1974) 111-128.

[14] H.K. Moffatt, Viscous and resistive eddies near a sharp corner, J. Fluid Mech. 18 (1964) 1-18.

[15] A. Acrivos, M.L. Schrader, Steady flow in a sudden expansion at high Reynolds number, J. Phys. Fluids 25 (1982) 923-930.

[16] F.S. Milos, A. Acrivos, J. Kim, Steady flow past sudden expansion at large Reynolds number-II. Navier-Stokes solutions for the cascade expansion, J. Phys. Fluids 30 (1987) 7-18.

[17] P. Towsend, K. Walters, Expansion flows of non-Newtonian liquids, Chem. Eng. Sci. 49 (1994) 749-763. 
[18] A. Baloch, P. Townsend, M.F. Webster, On two- and three-dimensional expansion flows, Computers and Fluids 24 (1995) 863-882.

[19] A. Baloch, P. Townsend, M.F. Webster, On vortex development in viscoelastic expansion and contraction flows, J. Non-Newt. Fluid Mech. 65 (1996) 133-149.

[20] N. Phan-Thien, R.I. Tanner, A new constitutive equation derived from network theory, J. Non-Newt. Fluid Mech. 2 (1977) 353-365.

[21] N. Phan-Thien, A non-linear network viscoelastic model, J. Rheol. 22 (1978) 259-283.

[22] A.L. Halmos, D.V. Boger, Flow of viscoelastic polymer solutions through an abrupt 2-to-1 expansion, Trans. Soc. Rheol. 20 (1976) 253-264.

[23] R.J. Poole, M.A. Alves, P.J. Oliveira, F.T. Pinho, Plane sudden expansion flows of viscoelastic liquids, J. Non-Newt. Fluid Mech. 146 (2007) 79-91.

[24] R.J. Poole, F.T. Pinho, M.A. Alves, P.J. Oliveira, The effect of expansion ratio for creeping expansion flows of UCM fluids, J. Non-Newt. Fluid Mech. 163 (2009) 35-44.

[25] W. Cherdron, F. Durst, J.H. Whitelaw, Asymmetric flows and instabilities in symmetric ducts with sudden expansions, J. Fluid Mech. 84 (1978) 13-31.

[26] R.M. Fearn, T. Mullin, K.A. Cliffe, Nonlinear phenomena in a symmetric sudden expansion, J. Fluid Mech. 211 (1990) 595-608.

[27] R. Manica, A.L. De Bortoli, Simulation of sudden expansion flows for power-law fluids, J. Non-Newt. Fluid Mech. 121 (2004) 35-40.

[28] P. Neofytou, Transition to asymmetry of generalised Newtonian fluid flows through a symmetric sudden expansion, J. Non-Newt. Fluid Mech. 133 (2006) $132-140$.

[29] P.J. Oliveira, Asymmetric flows of viscoelastic fluids in symmetric planar expansion geometries, J. Non-Newt. Fluid Mech. 114 (2003) 33-63.

[30] G.N. Rocha, R.J. Poole, P.J. Oliveira, Bifurcation phenomema in viscoelastic flows through a symmetric 1:4 expansion, J. Non-Newt. Fluid Mech. 141 (2007) 1-17.

[31] M.S.N. Oliveira, L.E. Rodd, G.H. McKinley, M.A. Alves, Simulations of extensional flow in microrheometric devices, Microfluid Nanofluid 5 (2008) 809-826.

[32] C. Dales, M.P. Escudier, R.J. Poole, Asymmetry in the turbulent flow of a viscoelastic liquid through an axisymmetric sudden expansion, J. Non-Newt. Fluid Mech. 125 (2005) 61-70.
33] T. Mullin, J.R.T. Seddon, M.D. Mantle, A.J. Sederman, Bifurcation phenomena in the flow through a sudden expansion in a circular pipe, Phys. Fluids 21 (2009) 014106-014110.

[34] G. Burgos, N.A. Alexandrou, Flow development of Herschel-Bulkley fluids in sudden 3-D expansion, J. Rheol. 43 (1999) 485-498.

[35] N.A. Alexandrou, T.M. McGilvreay, G. Burgos, Steady Herschel-Bulkley fluid flow in three-dimensional expansions, J. Non-Newt. Fluid Mech. 100 (2001) 77-96.

[36] R.D. Keane, R.J. Adrian, Theory of cross-correlation analysis of PIV images, Appl Sci. Res. 49 (1992) 191-215.

[37] J. Dealy, D. Plazek, Time-temperature superposition - a users guide, Rheol. Bull. 78 (2009) 16-31.

[38] P.J. Oliveira, F.T. Pinho, G.A. Pinto, Numerical simulation of non-linear elastic flows with a general collocated finite-volume method, J. Non-Newt. Fluid Mech. 79 (1998) 1-43.

[39] P.J. Coates, R.C. Armstrong, R.A. Brown, Calculation of steady-state viscoelastic flow through axisymmetric contractions with the EEME formulation, J. NonNewt. Fluid Mech. 42 (1992) 141-188.

[40] P.J. Oliveira, A.I.P. Miranda, A numerical study of steady and unsteady viscoelastic flow past bounded cylinders, J. Non-Newt. Fluid Mech. 127 (2005) 51-66.

41] P.C. Sousa, P.M. Coelho, M.S.N. Oliveira, M.A. Alves, Effect of the contraction ratio upon viscoelastic fluid flow in three-dimensional square-square contractions, Chem. Eng. Sci. 66 (2011) 998-1009.

[42] M.A. Alves, P.J. Oliveira, F.T. Pinho, A convergent and universally bounded interpolation scheme for the treatment of advection, Int. J. Numer. Methods Fluids 41 (2003) 47-75.

[43] M.A. Alves, F.T. Pinho, P.J. Oliveira, Visualizations of Boger fluid flows in a 4:1 square-square contraction, AIChE J. 51 (2005) 2908-2922.

[44] M.A. Alves, F.T. Pinho, P.J. Oliveira, Viscoelastic flow in a 3D square/square contraction: visualizations and simulations, J. Rheol. 52 (2008) 1347-1368.

[45] J.P. Rothstein, G.H. McKinley, The axisymmetric contraction-expansion: the role of extensional rheology on vortex growth dynamics and the enhanced pressure drop, J. Fluid Mech. 98 (2001) 33-63.

[46] R.B. Bird, R.C. Armstrong, O. Hassager, Dynamics of polymeric liquids, (vol. 1) Fluid Dynamics, John Wiley \& Sons, New York, 1987. 